\title{
LINEAR AND CONTINUOUS OPERATORS ON KÖTHE-BOCHNER SPACES
}

\author{
Ion Chiţescu and Răzvan-Cornel Sfetcu*
}

\begin{abstract}
University of Bucharest, Faculty of Mathematics and Computer Science Str. Academiei 14, 010014, Bucharest, Romania; University Politehnica of Bucharest Str. Splaiul Independenţei 313, 060042, Bucharest, Romania; ionchitescu@yahoo.com

University of Bucharest, Faculty of Mathematics and Computer Science Str. Academiei 14, 010014, Bucharest, Romania; razvancornelsfetcu@gmail.com
\end{abstract}

\begin{abstract}
The Köthe-Bochner spaces $L_{\rho}(E)$ are the vector valued version of the scalar Köthe spaces $L_{\rho}$, which generalize the Lebesgue spaces $L^{p}$, the Orlicz spaces and many other functional spaces. In the present paper we study the linear and continuous operators $U: \mathcal{L}_{\rho}(E) \rightarrow F$, giving integral representations for them. These operators generate operators $V: \mathcal{L}_{\rho} \rightarrow \mathcal{L}(E, F)$ which we call "natural operators" and study here.
\end{abstract}

\section{Introduction}

We study the linear and continuous operators defined on Köthe-Bochner spaces. The scalar Köthe spaces $L_{\rho}$ (name suggested by Dieudonné) generalize the Lebesgue spaces $L^{p}$ and a lot of other spaces, e.g. Orlicz spaces, Lorentz spaces, Marcinkiewicz spaces a.s.o. Primarily they appeared in [13], followed by [12] (as sequence spaces). The general presentation (as spaces of classes of measurable functions) appeared in [15], followed by [16], [24], [17], [25] and [3]. The "vector valued" Köthe spaces are called Köthe-Bochner spaces, being a natural generalization of the spaces $L_{\rho}$. In the present paper we operate with a more general definition of the Köthe-Bochner spaces than that one used in the autoritative monograph [14], precluding some unnecessary conditions (see the final considerations of the paragraph "Preliminary facts" and our previous paper [4]).

The present paper is concerned with the study of linear and continuous operators on Köthe-Bochner spaces. Of course, various situations can occur. For instance, in [10], operators having as range a Köthe-Bochner space are studied. In [2], one studies operators having both the domain and the range the same Köthe-Bochner space, in connection with the property of being multiplicative. The reader can find interesting and very technical facts concerning operators having the domain or the range Köthe spaces, variable Lebesgue spaces $L^{p}$ or Köthe-Bochner spaces in (the selection is, of course, subjective) the following papers: [1] (smoothness problems), [5] (boundedness on variable Lebesgue spaces $L^{p}$ ), [18] (integral operators on Köthe spaces, invariance and commutativity), [19] (narrow operators), [20] (maximal Hardy-Littlewood operator on variable Lebesgue spaces $L^{p}$ ), [21] (geometric properties of operators on Köthe spaces), [22], [23] (generation of chaos by operators defined on a Köthe sequence space).

https://doi.org/10.5186/aasfm.2019.4454

2010 Mathematics Subject Classification: Primary 46E30, 46E40, 47B38, 47G10; Secondary 28A20, 54A20, 54C35, 54E25.

Key words: Köthe space, Köthe-Bochner space, linear and continuous operator, variation of a measure, semivariation of a measure.

*Corresponding author. 
We recognize the decisive influence of [7], where linear and continuous operators on $\mathcal{L}^{p}(X, \mu)$ are studied using $q$-variation and $q$-semivariation. Working for KötheBochner spaces we generalize these ideas and add new ideas and facts. The present paper continues our previous paper [4].

\section{Preliminary facts}

Throughout the paper: $\mathbf{N}=\{1,2, \ldots\}, \mathbf{R}_{+}=[0, \infty), \overline{\mathbf{R}}_{+}=[0, \infty]=\mathbf{R}_{+} \cup\{\infty\}$, $K=\mathbf{R}$ or $\mathbf{C}$. All sequences $\left(x_{n}\right)_{n \in \mathbf{N}}$ or $\left(x_{n}\right)_{n}$ will be indexed with $\mathbf{N}$. The double sequences $\left(x_{p n}\right)_{p, n}$ will be indexed with $\mathbf{N} \times \mathbf{N}$. For any non empty set $T$, we shall denote by $\mathcal{P}(T)$ the set of all subsets of $T$. If $A \subset T, \varphi_{A}: T \rightarrow K$ is the characteristic (indicator) function of $A$. For $f: T \rightarrow K$ (or $f: T \rightarrow \overline{\mathbf{R}}_{+}$, or $f: T \rightarrow X, X$ normed space), we define $\operatorname{supp}(f) \stackrel{\text { def }}{=}\{t \in T \mid f(t) \neq 0\}$.

Let $X$ be a normed space with norm \|\| and let $T$ be a non empty set. If $f: T \rightarrow X$, we define the function $|f|: T \rightarrow \mathbf{R}_{+}$given via $|f|(t)=\|f(t)\|$ for any $t \in T$. If $\varphi: T \rightarrow K$ is another function, we define $\varphi f: T \rightarrow X$ via $(\varphi f)(t)=\varphi(t) f(t)$ for any $t \in T$. In particular: if $\varphi$ is constant, $\varphi \equiv \alpha \in K$, we have the function $\alpha f$; if $f$ is constant, $f \equiv x \in X$, we have the function $\varphi x$.

Let $(X, p)$ be a seminormed space and $(Y,\|\|)$ a normed space. The vector space $\mathcal{L}(X, Y) \stackrel{\text { def }}{=}\{V: X \rightarrow Y \mid V$ is linear and continuous $\}$ becomes a normed space (even a Banach space in case $Y$ is Banach) with the (operator) norm $\|V\|_{o} \stackrel{\text { def }}{=} \sup \{\|V(x)\| \mid$ $x \in X, p(x) \leq 1\}$. If $Y=K, \mathcal{L}(X, K)=X^{\prime}$.

Define $\operatorname{Ker}(p) \stackrel{\text { def }}{=}\{x \in X \mid p(x)=0\}$ and $\widetilde{X} \stackrel{\text { def }}{=} X / \operatorname{Ker}(p)$ and obtain the associated normed space $(\widetilde{X},||||||)$, where, for any $\widetilde{x} \in \widetilde{X},|\|\widetilde{x} \mid\| \stackrel{\text { def }}{=} p(x)$ for all $x \in \widetilde{x}$ (coherent definition). Then $\widetilde{X}$ is Banach if and only if $(X, p)$ is a complete semimetric space. The normed spaces $\mathcal{L}(X, Y)$ and $\mathcal{L}(\widetilde{X}, Y)$ are linearly and isometrically isomorphic via the isomorphism $\Omega: \mathcal{L}(X, Y) \rightarrow \mathcal{L}(\widetilde{X}, Y)$, acting as follows: $\Omega(V)=\widetilde{V}$ (the associated operator of $V)$, where $\widetilde{V}(\widetilde{x}) \stackrel{\text { def }}{=} V(x)$ for any $\widetilde{x} \in \widetilde{X}$ and any $x \in \widetilde{x}$ (coherent definition). So, in order to study $\mathcal{L}(\widetilde{X}, Y)$, one can study $\mathcal{L}(X, Y)$. This procedure will be used in this paper, namely we shall study linear and continuous operators $V: \mathcal{L}_{\rho}(E) \rightarrow F$ instead of linear and continuous operators $V: L_{\rho}(E) \rightarrow F$.

If $X, E, F$ are Banach spaces, we say that $X$ is embedded in $\mathcal{L}(E, F)$ (and write $X \hookrightarrow \mathcal{L}(E, F))$ in case there exists a linear and isometric map $H: X \rightarrow \mathcal{L}(E, F)$ (so $\|x\|=\|H(x)\|_{o}$ for any $x \in X$ ). Two conventions are in use: a) Generally, one omits to explicitely mention the map $H$; b) There is total identification $x \equiv H(x)$ for any $x \in X$ (e.g. one writes either $\|x\|$ or $\|x\|_{o}$ to designate the norm of $x$ ). For any normed space one can find $E, F$ such that $X \hookrightarrow \mathcal{L}(E, F)$. The most popular embeddings are the following two canonical embeddings: a) $X \hookrightarrow \mathcal{L}(K, X) \equiv X$, with $H(x)=T_{x}, T_{x}(\alpha)=\alpha x$ for any $x \in X, \alpha \in K$; b) $X \hookrightarrow \mathcal{L}\left(X^{\prime}, K\right)=X^{\prime \prime}$, with $H(x)=V_{x}, V_{x}\left(x^{\prime}\right)=x^{\prime}(x)$ for any $x \in X, x^{\prime} \in X^{\prime}$.

For supplementary functional analysis facts, one can consult [8]. For vector measures and integration, the standard texts are [6] and [7].

In the sequel we shall present the Köthe-Bochner spaces. We shall work with a $\sigma$-finite and complete measure space $(T, \mathcal{T}, \mu)$, i.e. $T$ is a non empty set, $\mathcal{T} \subset \mathcal{P}(T)$ is a $\sigma$-algebra of sets and $\mu: \mathcal{T} \rightarrow \overline{\mathbf{R}}_{+}$is a measure which is $\sigma$-finite and complete. For general measure theory, see [11]. In particular, we shall also deal with the countable discrete measure space $(\mathbf{N}, \mathcal{P}(\mathbf{N})$, card) or with the finite discrete measure space $(T, \mathcal{P}(T)$, card $)$, where $\phi \neq T$ is finite and $\operatorname{card}(A)=$ the number of elements in $A$, if 
$A$ is finite and $\operatorname{card}(A)=\infty$, if $A$ is infinite. For Köthe spaces see [3] and [24]. For Köthe-Bochner spaces see [4] and [14].

The set of all $\mu$-measurable positive functions $u: T \rightarrow \overline{\mathbf{R}}_{+}$will be denoted by $M_{+}(\mu)$. A $\mu$-function norm (or, simply, a function norm) is a function $\rho: M_{+}(\mu) \rightarrow$ $\overline{\mathbf{R}}_{+}$having the following properties (for any $u, v$ in $M_{+}(\mu)$ and any $\alpha \in \mathbf{R}_{+}$): 1) $\rho(u)=0$ if and only if $u(t)=0 \mu$-a.e.; 2) $\rho(u) \leq \rho(v)$, whenever $u \leq v$; 3) $\rho(u+v) \leq \rho(u)+\rho(v)$; 4) $\rho(\alpha u)=\alpha \rho(u)$, with the convention $0 \cdot \infty=0$. For instance, we can consider the particular example of the Lebesgue function norms \|\|$_{p}, 1 \leq p \leq \infty$.

For any $A \in \mathcal{T}$, we write $\rho(A) \stackrel{\text { def }}{=} \rho\left(\varphi_{A}\right)$. We shall write $\mathcal{T}_{\rho}=\{A \in \mathcal{T} \mid \rho(A)<$ $\infty$ \}. It is seen that $\mathcal{T}_{\rho}$ is a $\delta$-ring (semitribe).

We say that the function norm $\rho$ has the Riesz-Fischer property if $\rho\left(\sum_{n=1}^{\infty} u_{n}\right) \leq$ $\sum_{n=1}^{\infty} \rho\left(u_{n}\right)$ for any sequence $\left(u_{n}\right)_{n} \subset M_{+}(\mu)$. In particular, all the function norms \|\|$_{p}, 1 \leq p \leq \infty$ have this property.

For any function norm $\rho$ one can construct the associated function (semi)norm $\rho^{\prime}$ of $\rho$. Namely $\rho^{\prime}: M_{+}(\mu) \rightarrow \overline{\mathbf{R}}_{+}$acts via $\rho^{\prime}(u)=\sup \left\{\int u v d \mu \mid v \in M_{+}(\mu), \rho(v) \leq 1\right\}$. We feel obliged to remind the fact that in some pathological cases, it is possible to have $\rho^{\prime}(u)=0$, even in case the assertion $u(t)=0 \mu$-a.e. is not true. If $\rho=\|\|_{p}$, $1 \leq p \leq \infty$, we have $\rho^{\prime}=\|\|_{q}$, where $q$ is the conjugate of $p$, i.e. $\frac{1}{p}+\frac{1}{q}=1$ with the conventions $\frac{1}{\infty}=0$ and $\frac{1}{0}=\infty$.

We say that the function norm $\rho$ is of absolutely continuous type if it has the following property: for any $u \in M_{+}(\mu)$ with $\rho(u)<\infty$ and for any decreasing sequence $\left(u_{n}\right)_{n} \subset M_{+}(\mu)$ such that $u_{n} \leq u \mu$-a.e. for any $n$ and such that $\lim _{n} u_{n}(t)=$ $0 \mu$-a.e. (we write $u_{n} \downarrow 0$ ), we have $\rho\left(u_{n}\right) \underset{n}{\rightarrow} 0$ (we write $\rho\left(u_{n}\right) \downarrow 0$ ). All \|\|$_{p}$, $1 \leq p<\infty$, are of absolutely continuous type. Notice that, when working with the finite discrete measure space, any finite function norm is of absolutely continuous type (because all norms on a finite dimensional vector space are equivalent and the convergence is normic).

Now, let us consider a Banach space $X$. We shall denote by $M_{X}(\mu)$ the vector space of all $\mu$-measurable functions $f: T \rightarrow X$. Let also $N_{X}(\mu)$ the vector space of all (of course $\mu$-measurable) functions $f: T \rightarrow X$ having the property that $f(t)=0$ $\mu$-a.e. For any $f \in M_{X}(\mu)$, write $\rho|f| \stackrel{\text { def }}{=} \rho(|f|)$. Define the vector space

$$
\mathcal{L}_{\rho}(X)=\left\{f \in M_{X}(\mu)|\rho| f \mid<\infty\right\}
$$

which is a seminormed space with the seminorm $f \longmapsto p(f) \stackrel{\text { def }}{=} \rho|f|$. Then $\operatorname{Ker}(p)=$ $N_{X}(\mu)$ and we obtain the associated normed space

$$
L_{\rho}(X) \stackrel{\text { def }}{=} \mathcal{L}_{\rho}(X) / N_{X}(\mu),
$$

normed with the norm $\tilde{f} \longmapsto\|\tilde{f}\|=\rho|f|$ for any $f \in \tilde{f}$.

We call $\mathcal{L}_{\rho}(X)$ and $L_{\rho}(X)$ Köthe-Bochner spaces. In case $X=K$, we write only $\mathcal{L}_{\rho}\left(\right.$ instead of $\left.\mathcal{L}_{\rho}(K)\right)$ and $L_{\rho}\left(\right.$ instead of $\left.L_{\rho}(K)\right)$ and call $\mathcal{L}_{\rho}$ and $L_{\rho}$ Köthe spaces. So $\mathcal{L}_{\rho}(X)=\left\{f \in M_{X}(\mu)|| f \mid \in \mathcal{L}_{\rho}\right\}$. It is known that $\mathcal{L}_{\rho}(X)$ (respectively $\left.L_{\rho}(X)\right)$ is complete if and only if $\rho$ has the Riesz-Fischer property (see [4]).

In case $\rho=\|\|_{p}, 1 \leq p \leq \infty$, we have, instead of $\mathcal{L}_{\rho}(X)$ and $L_{\rho}(X)$, the Lebesgue spaces $\mathcal{L}^{p}(X, \mu)$ and $L^{p}(X, \mu)$ (respectively $\mathcal{L}^{p}(\mu)$ and $L^{p}(\mu)$, if $\left.X=K\right)$.

If $(T, \mathcal{T}, \mu)$ is the countable (respectively finite) discrete measure space $(\mathbf{N}, \mathcal{P}(\mathbf{N})$, card) (respectively $(T=\{1,2, \ldots, n\}, \mathcal{P}(T)$, card)), the only negligible set is $\phi$, 
all functions (sequences or $n$-tuples) are measurable and $\mathcal{L}_{\rho}(X) \equiv L_{\rho}(X)$. For the countable case, we write $l_{\rho}(X)$ instead of $\mathcal{L}_{\rho}(X), l_{\rho}$ instead of $\mathcal{L}_{\rho}, l^{p}(X)$ instead of $\mathcal{L}^{p}(X$, card $), l^{p}$ instead of $\mathcal{L}^{p}$ (card). For the finite case, we have $\mathcal{L}_{\rho}(X)=$ $\mathcal{L}^{p}(X$, card $) \equiv X^{n}$ and $\mathcal{L}_{\rho}=\mathcal{L}^{p}($ card $) \equiv K^{n}$ (assuming also that $\rho$ takes only finite values).

Returning to a general measure space $(T, \mathcal{T}, \mu)$ we introduce some special notations which will be used further. Let $E$ be a Banach space. A function $f: T \rightarrow E$ will be called $\rho$-simple if it has the form $f=\sum_{i=1}^{n} \varphi_{A_{i}} x_{i}$, where $A_{i} \in \mathcal{T}_{\rho}$ are mutually disjoint sets and all $x_{i} \in E$. The set of all $\rho$-simple functions will be denoted with $S_{E}(\rho)$. Clearly $S_{E}(\rho) \subset \mathcal{L}_{\rho}(E)$. If $E=K$ we write only $S(\rho)$. For any $A \subset T$, write $S_{E}(\rho, A)=\left\{f \in S_{E}(\rho) \mid \operatorname{supp}(f) \subset A\right\}$ (if $E=K$, write only $S(\rho, A)$ ). Write also $S_{E}(\rho, 1)=\left\{f \in S_{E}(\rho)|\rho| f \mid \leq 1\right\}$ (if $E=K$, write only $S(\rho, 1)$ ). We can consider also $S_{E}(\rho, A, 1)=\left\{f \in S_{E}(\rho, A)|\rho| f \mid \leq 1\right\}$ (if $E=K$, write only $S(\rho, A, 1)$ ).

The following basic result will be fundamental for our further study. We give it here with proof, in order to make our paper to be self-contained.

Density Lemma. If $\rho$ is of absolutely continuous type, $S_{E}(\rho)$ is dense in $\mathcal{L}_{\rho}(E)$.

Proof. We consider an arbitrary $f \in \mathcal{L}_{\rho}(E)$ and show how to construct a sequence $\left(f_{n}\right)_{n} \subset S_{E}(\rho)$ such that $f_{n} \underset{n}{\rightarrow} f$ in $\mathcal{L}_{\rho}(E)$.

Indeed, for the $\mu$-measurable $f$, one can find a sequence $\left(f_{n}\right)_{n} \subset S_{E}(\rho)$ such that $\left(f_{n}\right)_{n}$ converges to $f \mu$-a.e. and $\left|f_{n}\right| \leq|f| \mu$-a.e. for any $n$ (see [7]). Define, for any $n$, the function $u_{n}: T \rightarrow \mathbf{R}_{+}$via $u_{n}(t)=\sup _{m}\left\|f_{n+m}(t)-f(t)\right\| \leq 2|f|(t)$. Then $u_{n} \downarrow 0$ $\mu$-a.e. Because $\rho$ is of absolutely continuous type, it follows that $\rho\left(u_{n}\right) \downarrow 0$. Clearly, $\left|f_{n+1}-f\right| \leq u_{n}$, hence $\rho\left|f_{n}-f\right| \underset{n}{\rightarrow} 0$ and $f_{n} \underset{n}{\rightarrow} f$ in $\mathcal{L}_{\rho}(E)$.

Remarks. 1. The present paper differs from the paper [9] which is concerned with the same subject: many conditions imposed in [9] do not appear here and the results (obtained with other methods) are expressed in a different manner. To be more specific: A. The exposition in [9] relies heavily upon the conditional expectation generated by a partition (actually, this name does not specifically appear in [9]) and upon admissible sequences. In our paper, we do not speak about these types of facts. B. The function norm $\rho$ is assumed to possess the weak Fatou property, stronger than the Riesz-Fischer property (which implies the completeness of the Köthe-Bochner spaces $L_{\rho}(X)$ ) and the so-called property $(J)$ (related to the conditional expectation generated by a partition). These two properties are not assumed in the present paper.

2. In the authoritative monograph [14], the space $\mathcal{L}_{\rho}$ is defined adding the following supplementary conditions: a) $K=\mathbf{R}$; b) The functions in $\mathcal{L}_{\rho}$ must be locally $\mu$-integrable; c) For any $A \in \mathcal{T}$ with $\mu(A)<\infty$ one has $\varphi_{A} \in \mathcal{L}_{\rho}$; d) $\mathcal{L}_{\rho}$ is Banach. We do not impose these conditions in our paper (see also our previous paper [4]). We lay stress upon the fact that the lastly imposed condition d) is extreme, excluding from the study a lot of $\mathcal{L}_{\rho}$ spaces. Linear and continuous operators on Köthe-Bochner spaces are not studied in [14].

\section{Generalization of variation and semivariation}

Let $(T, \mathcal{T}, \mu)$ be a $\sigma$-finite and complete measure space and $\rho: M_{+}(\mu) \rightarrow \overline{\mathbf{R}}_{+}$ a function norm. We shall consider three Banach spaces $X, E, F$ such that $X \hookrightarrow$ $\mathcal{L}(E, F)$. Let $m: \mathcal{T}_{\rho} \rightarrow X$ be a function with the property $m(\phi)=0$ (in particular, $m$ can be additive).

Definition 3.1. (Generalization of classic notions) Let $A \subset T$. 
1. The $(\rho,(E, F))$-variation of $m$ on $A$ is the element $\bar{m}_{\rho,(E, F)}(A) \in \overline{\mathbf{R}}_{+}$defined via

$$
\bar{m}_{\rho,(E, F)}(A)=\sup \left\{\sum_{i=1}^{n}\left\|m\left(A_{i}\right)\left(x_{i}\right)\right\| \mid \sum_{i=1}^{n} \varphi_{A_{i}} x_{i} \in S_{E}(\rho, A, 1)\right\} .
$$

2. The $(\rho,(E, F))$-semivariation of $m$ on $A$ is the element $\widetilde{m}_{\rho,(E, F)}(A) \in \overline{\mathbf{R}}_{+}$ defined via

$$
\widetilde{m}_{\rho,(E, F)}(A)=\sup \left\{\left\|\sum_{i=1}^{n} m\left(A_{i}\right)\left(x_{i}\right)\right\| \mid \sum_{i=1}^{n} \varphi_{A_{i}} x_{i} \in S_{E}(\rho, A, 1)\right\} .
$$

Remark. Clearly, for any embedding $X \hookrightarrow \mathcal{L}(E, F)$ and any $A \subset T$, one has $\widetilde{m}_{\rho,(E, F)}(A) \leq \bar{m}_{\rho,(E, F)}(A)$.

We shall see that $\bar{m}_{\rho,(E, F)}(A)$ does not depend upon the embedding $X \hookrightarrow \mathcal{L}(E, F)$, being the same for all embeddings.

Theorem 3.2. (Invariance of the variation with respect to the embedding) For any embedding $X \hookrightarrow \mathcal{L}(E, F)$ and any $A \subset T$ one has $\bar{m}_{\rho,(E, F)}(A)=\bar{m}_{\rho,(K, X)}(A)=$ $\sup \left\{\sum_{i=1}^{n}\left|\alpha_{i}\right|\left\|m\left(A_{i}\right)\right\| \mid \sum_{i=1}^{n} \varphi_{A_{i}} \alpha_{i} \in S(\rho, A, 1)\right\}\left(\bar{m}_{\rho,(K, X)}(A)\right.$ is computed for the canonical embedding (isomorphism) $X \equiv \mathcal{L}(K, X)$ ).

Proof. 1. Take arbitrarily $\sum_{i=1}^{n} \varphi_{A_{i}} x_{i} \in S_{E}(\rho, A, 1)$. Hence $\sum_{i=1}^{n} \varphi_{A_{i}}\left\|x_{i}\right\| \in$ $S(\rho, A, 1)$ and $\sum_{i=1}^{n}\left\|m\left(A_{i}\right)\left(x_{i}\right)\right\| \leq \sum_{i=1}^{n}\left\|m\left(A_{i}\right)\right\|_{o}\left\|x_{i}\right\|$, i.e. $\bar{m}_{\rho,(E, F)}(A) \leq \bar{m}_{\rho,(K, X)}(A)$ because $m(A)(\alpha)=\alpha m(A)$ for any $\alpha \in K$ and $X \equiv \mathcal{L}(K, X)$.

2. In order to prove the converse inequality, take $\sum_{i=1}^{n} \varphi_{A_{i}} \alpha_{i} \in S(\rho, A, 1)$. Let $\varepsilon>$ 0 . For any $i=1,2, \ldots, n$, one has $\left|\alpha_{i}\right|\left\|m\left(A_{i}\right)\right\|_{o}=\left\|\alpha_{i} m\left(A_{i}\right)\right\|_{o}=\sup \left\{\left\|m\left(A_{i}\right)\left(\alpha_{i} y\right)\right\| \mid\right.$ $y \in E,\|y\|=1\}$, hence one can find $y_{i} \in E,\left\|y_{i}\right\|=1$ such that $\left|\alpha_{i}\right|\left\|m\left(A_{i}\right)\right\|_{o}-\frac{\varepsilon}{n} \leq$ $\left\|m\left(A_{i}\right)\left(\alpha_{i} y_{i}\right)\right\|$, hence

$$
\sum_{i=1}^{n}\left|\alpha_{i}\right|\left\|m\left(A_{i}\right)\right\|_{o}-\varepsilon \leq \sum_{i=1}^{n}\left\|m\left(A_{i}\right)\left(x_{i}\right)\right\|,
$$

with $x_{i} \stackrel{\text { def }}{=} \alpha_{i} y_{i}$. Define $f=\sum_{i=1}^{n} \varphi_{A_{i}} x_{i}$. We have $|f|=\sum_{i=1}^{n} \varphi_{A_{i}}\left|\alpha_{i}\right| \Rightarrow f \in$ $S_{E}(\rho, A, 1) \Rightarrow \sum_{i=1}^{n}\left\|m\left(A_{i}\right)\left(x_{i}\right)\right\| \leq \bar{m}_{\rho,(E, F)}(A)$ and (3.1) implies $\sum_{i=1}^{n}\left|\alpha_{i}\right|\left\|m\left(A_{i}\right)\right\|_{o}$ $-\varepsilon \leq \bar{m}_{\rho,(E, F)}(A)$. Passing to supremum in the left-hand side of the last inequality, we get $\bar{m}_{\rho,(K, X)}(A)-\varepsilon \leq \bar{m}_{\rho,(E, F)}(A)$, hence $\bar{m}_{\rho,(K, X)}(A) \leq \bar{m}_{\rho,(E, F)}(A)$, because $\varepsilon$ is arbitrary, a.s.o.

Notation. According to the preceding result, we shall write $\bar{m}_{\rho}(A) \stackrel{\text { def }}{=} \bar{m}_{\rho,(E, F)}(A)$ for any embedding $X \hookrightarrow \mathcal{L}(E, F)$ and we shall call $\bar{m}_{\rho}(A)$ the $\rho$-variation of $m$ on A.

Definition 3.3. In view of the aforementioned facts, we see that, for any function norm $\rho$ and any embedding $X \hookrightarrow \mathcal{L}(E, F)$, we have defined the functions $\widetilde{m}_{\rho,(E, F)}: \mathcal{P}(T) \rightarrow \overline{\mathbf{R}}_{+}$(respectively $\bar{m}_{\rho}: \mathcal{P}(T) \rightarrow \overline{\mathbf{R}}_{+}$) via $A \longmapsto \widetilde{m}_{\rho,(E, F)}(A)$ (respectively $\left.A \longmapsto \bar{m}_{\rho}(A)\right)$.

Example 3.4. We shall exhibit situations when the general inequality $\widetilde{m}_{\rho,(E, F)}(A)$ $\leq \bar{m}_{\rho}(A)$ is either strict, or an equality. The examples will be given for $T=[0,1]$, $\mathcal{T}=$ the Lebesgue measurable sets of $[0,1]$ and $\mu=$ the Lebesgue measure on $[0,1]$. The function norm will be $\rho=\|\|_{p}, 1 \leq p \leq \infty$. We shall work for $X=L_{\rho}$, i.e. $X=L^{p}(\mu)$ and for the canonical embedding (isomorphism) $X \equiv \mathcal{L}(K, X)$. Finally $m: \mathcal{T}_{\rho} \rightarrow X$ is given via $m(A)=\widetilde{\varphi}_{A}(\sigma$-additive for $p<\infty$ and additive for $p=\infty)$. 
1. For $p=1$ (i.e. $\rho=\|\|_{1}$ ), one has $\bar{m}_{\rho}(T)=\widetilde{m}_{\rho,(K, X)}(T)=\widetilde{m}_{\rho,(E, F)}(T)=1$ for any $X \hookrightarrow \mathcal{L}(E, F)$ (see also Theorem 3.5).

2. For $1<p \leq \infty$ (i.e. $\rho=\|\|_{p}$ ), one has $\widetilde{m}_{\rho,(K, X)}(T)=1<\bar{m}_{\rho}(T)=\infty$.

Sketch of the proof. 1. We have $\bar{m}_{\rho}(T)=1$, because $\rho(1)=1$ and, for $\varphi \in$ $S\left(\|\|_{1}, 1\right), \varphi=\sum_{i=1}^{n} \alpha_{i} \varphi_{A_{i}}$, one has $\sum_{i=1}^{n}\left|\alpha_{i}\right|\left\|m\left(A_{i}\right)\right\|_{1}=\rho|\varphi| \leq 1$.

We have $\tilde{m}_{\rho,(K, X)}(T)=1$, because $\rho(1)=1$ and, for $\varphi \in S\left(\|\|_{1}, 1\right), \varphi=$ $\sum_{i=1}^{n} \alpha_{i} \varphi_{A_{i}}$, one has $\left\|\sum_{i=1}^{n} m\left(A_{i}\right)\left(\alpha_{i}\right)\right\|_{1}=\rho|\varphi| \leq 1$.

2. The idea is to express, for any $n \in \mathbf{N}$, the constant function $\varphi \equiv 1, \varphi \in$ $S(\rho, 1)$, as follows: $\varphi=\sum_{i=1}^{n} \varphi_{A_{i}^{n}} \alpha_{i}^{n}$, where all $\alpha_{i}^{n}=1$ and $A_{i}^{n}=\left[\frac{i-1}{n}, \frac{i}{n}\right)$, for $i=1,2, \ldots, n-1$ and $A_{n}^{n}=\left[\frac{n-1}{n}, 1\right]$.

We have $\widetilde{m}_{\rho,(K, X)}(T)=1$ in all cases (same proof as at point 1.).

We have $\bar{m}_{\rho}(T)=\infty$ in all cases, considering the above mentioned expression of $\varphi \equiv 1$. Namely, for $1<p<\infty, \sum_{i=1}^{n}\left\|\alpha_{i}^{n} m\left(A_{i}^{n}\right)\right\|_{p}=\sum_{i=1}^{n}\left\|m\left(A_{i}^{n}\right)\right\|_{p}=n \cdot\left(\frac{1}{n}\right)^{\frac{1}{p}}=$ $n^{1-\frac{1}{p}} \underset{n}{\rightarrow} \infty$. For $p=\infty, \sum_{i=1}^{n}\left\|\alpha_{i}^{n} m\left(A_{i}^{n}\right)\right\|_{\infty}=\sum_{i=1}^{n}\left\|m\left(A_{i}^{n}\right)\right\|_{\infty}=n \underset{n}{\rightarrow} \infty$.

Considering the values of $\widetilde{m}_{\rho,(E, F)}$ for different embeddings $X \hookrightarrow \mathcal{L}(E, F)$, we obtain, for any $m: \mathcal{T}_{\rho} \rightarrow X$ with $m(\phi)=0$

Theorem 3.5. $\quad$ 1. $\widetilde{m}_{\rho,(K, X)} \leq \widetilde{m}_{\rho,(E, F)}$.

2. In case $F=K$, i.e. $X \hookrightarrow E^{\prime}=\mathcal{L}(E, K): \widetilde{m}_{\rho,(E, K)}=\bar{m}_{\rho}$.

3. Consequently: $\widetilde{m}_{\rho,(K, X)} \leq \widetilde{m}_{\rho,(E, F)} \leq \widetilde{m}_{\rho,\left(X^{\prime}, K\right)}=\bar{m}_{\rho}$.

Proof. Let $A \subset T$.

1. Considering $\varphi=\sum_{i=1}^{n} \alpha_{i} \varphi_{A_{i}} \in S(\rho, A, 1)$, we have $\left\|\sum_{i=1}^{n} \alpha_{i} m\left(A_{i}\right)\right\|_{o}=$ $\sup _{\|x\| \leq 1}\left\|\sum_{i=1}^{n} \alpha_{i} m\left(A_{i}\right)(x)\right\|=\sup _{\|x\| \leq 1}\left\|\sum_{i=1}^{n} m\left(A_{i}\right)\left(\alpha_{i} x\right)\right\| \leq \widetilde{m}_{\rho,(E, F)}(A)$, because $\sum_{i=1}^{n} \varphi_{A_{i}} \alpha_{i} x \in S_{E}(\rho, A, 1)$, if $x \in E,\|x\| \leq 1$. We pass to supremum with respect to $\varphi$.

2. Use the equalities

$$
\bar{m}_{\rho}(A)=\bar{m}_{\rho,(K, X)}(A)=\sup \left\{\sum_{i=1}^{n}\left|\alpha_{i}\right|\left\|m\left(A_{i}\right)\right\|_{o} \mid \sum_{i=1}^{n} \varphi_{A_{i}} \alpha_{i} \in S(\rho, A, 1)\right\} .
$$

Then, for any $\varphi=\sum_{i=1}^{n} \varphi_{A_{i}} \alpha_{i} \in S(\rho, A, 1)$, any $i=1,2, \ldots, n$ and any $\varepsilon>0$, we find $x_{i} \in E$ with $\left\|x_{i}\right\|=1$ such that

$$
\left|\alpha_{i}\right|\left\|m\left(A_{i}\right)\right\|_{o}=\left\|\alpha_{i} m\left(A_{i}\right)\right\|_{o}<\left|\left(\alpha_{i} m\left(A_{i}\right)\right)\left(x_{i}\right)\right|+\frac{\varepsilon}{n}=m\left(A_{i}\right)\left(\alpha_{i} x_{i}\right)+\frac{\varepsilon}{n} .
$$

It is seen that $\left|\sum_{i=1}^{n} \varphi_{A_{i}} \alpha_{i} x_{i}\right|=|\varphi|$, hence $\sum_{i=1}^{n} \varphi_{A_{i}} \alpha_{i} x_{i} \in S_{E}(\rho, A, 1)$. Consequently, $\sum_{i=1}^{n}\left|\alpha_{i}\right|\left\|m\left(A_{i}\right)\right\|_{o}<\sum_{i=1}^{n} m\left(A_{i}\right)\left(\alpha_{i} x_{i}\right)+\varepsilon=\left|\sum_{i=1}^{n} m\left(A_{i}\right)\left(\alpha_{i} x_{i}\right)\right|+\varepsilon \leq$ $\widetilde{m}_{\rho,(E, K)}(A)+\varepsilon$. Because $\varphi \in S(\rho, A, 1)$ is arbitrary, we get $\bar{m}_{\rho}(A) \leq \widetilde{m}_{\rho,(E, K)}(A)+\varepsilon$. But $\varepsilon>0$ is arbitrary, hence $\bar{m}_{\rho}(A) \leq \widetilde{m}_{\rho,(E, K)}(A)$ a.s.o.

The following four properties will be used further, especially to prove Proposition 3.6, Proposition 3.7 and Theorem 3.8.

Property 1. $\widetilde{m}_{\rho,(E, F)}(\phi)=\bar{m}_{\rho}(\phi)=0$.

Property 2. For $A \subset B \subset T, \widetilde{m}_{\rho,(E, F)}(A) \leq \widetilde{m}_{\rho,(E, F)}(B)$ and $\bar{m}_{\rho}(A) \leq \bar{m}_{\rho}(B)$.

Property 3. If $A \subset T:\left(\bar{m}_{\rho}(A)=0\right) \Leftrightarrow\left(\widetilde{m}_{\rho,(E, F)}(A)=0\right) \Leftrightarrow\left(\right.$ For any $\mathcal{T}_{\rho} \ni B \subset$ $A$, one has $m(B)=0)$. In particular, if $A \in \mathcal{T}_{\rho}:\left(\bar{m}_{\rho}(A)=0\right) \Rightarrow(m(A)=0)$.

Property 4. Let $A \in \mathcal{T}_{\rho}$.

a) $(\mu(A)=0$ and $m(A) \neq 0) \Rightarrow\left(\mu(A)=0\right.$ and $\left.\widetilde{m}_{\rho,(E, F)}(A)=\bar{m}_{\rho}(A)=\infty\right)$. 
b) Assume $\mu(A)=0$. Then: i) $\left(\widetilde{m}_{\rho,(E, F)}(A)<\infty\right) \Leftrightarrow\left(\widetilde{m}_{\rho,(E, F)}(A)=0\right)$; ii) $\left(\bar{m}_{\rho}(A)<\infty\right) \Leftrightarrow\left(\bar{m}_{\rho}(A)=0\right)$.

Proposition 3.6. Let $A \subset T$ and $f=\sum_{i=1}^{n} \varphi_{A_{i}} x_{i} \in S_{E}(\rho, A)$. Assume that either $\rho|f| \neq 0$, or $\widetilde{m}_{\rho,(E, F)}(A)<\infty$. Then: $\left.a\right)\left\|\sum_{i=1}^{n} m\left(A_{i}\right)\left(x_{i}\right)\right\| \leq \widetilde{m}_{\rho,(E, F)}(A) \rho|f|$; b) $\sum_{i=1}^{n}\left\|m\left(A_{i}\right)\right\|_{o}\left\|x_{i}\right\| \leq \bar{m}_{\rho}(A) \rho|f|$.

Proof. We prove $a$ ), the proof of $b$ ) being similar. Assume first $\rho|f| \neq 0$, hence $\sum_{i=1}^{n} \frac{\varphi_{A_{i}}}{\rho|f|} x_{i} \in S_{E}(\rho, A, 1) \Rightarrow\left\|\frac{1}{\rho|f|} \sum_{i=1}^{n} m\left(A_{i}\right)\left(x_{i}\right)\right\| \leq \widetilde{m}_{\rho,(E, F)}(A)$

In case $\rho|f|=0$, we have $\widetilde{m}_{\rho,(E, F)}(A)<\infty$ and, for any $i, \rho\left|\varphi_{A_{i}} x_{i}\right|=\left\|x_{i}\right\| \rho\left(A_{i}\right)$ $\leq \rho|f|=0$, hence either $x_{i}=0$, or $\rho\left(A_{i}\right)=0$. We shall see that one has $m\left(A_{i}\right)\left(x_{i}\right)=0$ for any $n$. Indeed, if $x_{i} \neq 0$, we have $\rho\left(A_{i}\right)=0$, hence $\mu\left(A_{i}\right)=0$. We also have $\widetilde{m}_{\rho,(E, F)}\left(A_{i}\right) \leq \widetilde{m}_{\rho,(E, F)}(A)<\infty$ (Property 2) and this implies $\widetilde{m}_{\rho,(E, F)}\left(A_{i}\right)=0$ (Property 4). Using Property 3 we get $m\left(A_{i}\right)=0$, hence $m\left(A_{i}\right)\left(x_{i}\right)=0$. It follows that $\sum_{i=1}^{n} m\left(A_{i}\right)\left(x_{i}\right)=0$ a.s.o.

Proposition 3.7. Let $A \in \mathcal{T}_{\rho}$. Consider the propositions: $P_{1}$ : Either $\mu(A) \neq 0$, or $m(A)=0 ; P_{2}$ : Either $\mu(A) \neq 0$, or $\widetilde{m}_{\rho,(E, F)}(A)<\infty$. Then $P_{i} \Rightarrow\left(\|m(A)\|_{o} \leq\right.$ $\left.\widetilde{m}_{\rho,(E, F)}(A) \rho(A) \leq \bar{m}_{\rho}(A) \rho(A)\right)(i=1,2)$.

Proof. First, we prove that $P_{1} \Rightarrow\left(\|m(A)\|_{o} \leq \widetilde{m}_{\rho,(E, F)}(A) \rho(A)\right)$, in the non trivial case $\mu(A) \neq 0$ (if $\mu(A)=0$, we have $m(A)=0$ ). Take $0 \neq x \in E$. Then $f=\varphi_{A} x \in S_{E}(\rho, A)$ with $\rho|f|=\rho(A)\|x\| \neq 0$. Proposition 3.6 implies $\|m(A)(x)\| \leq$ $\widetilde{m}_{\rho,(E, F)}(A) \rho|f|=\widetilde{m}_{\rho,(E, F)}(A) \rho(A)\|x\|$, hence $\|m(A)\|_{o} \leq \widetilde{m}_{\rho,(E, F)}(A) \rho(A)$. It remains to prove $P_{2} \Rightarrow\left(\|m(A)\|_{o} \leq \widetilde{m}_{\rho,(E, F)}(A) \rho(A)\right)$, in case $\mu(A)=0$. Because $\widetilde{m}_{\rho,(E, F)}(A)<\infty$, we have (Property 4. b)) that $\widetilde{m}_{\rho,(E, F)}(A)=0$ and this implies $m(A)=0$ (Property 3.).

Theorem 3.8. Assume that $\rho$ is of absolutely continuous type, $m: \mathcal{T}_{\rho} \rightarrow X \hookrightarrow$ $\mathcal{L}(E, F)$ is additive and $\widetilde{m}_{\rho,(E, F)}(A)<\infty$ for any $A \in \mathcal{T}_{\rho}$. Then $m$ is $\sigma$-additive and $m$ is locally absolutely continuous with respect to $\mu$, i.e. for any $A \in \mathcal{T}_{\rho}$ and any $\varepsilon>0$, there exists $\delta_{A}>0$ such that (For any $A \supset B \in \mathcal{T}_{\rho}$ and $\left.\mu(B)<\delta_{A}\right) \Rightarrow(\|m(B)\|<\varepsilon)$.

Proof. Take a decresing sequence $\left(A_{n}\right)_{n} \subset \mathcal{T}_{\rho}$ such that $A_{n} \downarrow \phi$ (i.e. $\bigcap_{n=1}^{\infty} A_{n}=$ $\phi)$. Then $\varphi_{A_{n}} \downarrow 0$ and this implies $\rho\left(A_{n}\right) \downarrow 0$, because $\rho$ is of absolutely continuous type. Proposition 3.7 and Property 2. say that $\left\|m\left(A_{n}\right)\right\|_{o}=\left\|m\left(A_{n}\right)\right\| \leq$ $\widetilde{m}_{\rho,(E, F)}\left(A_{n}\right) \rho\left(A_{n}\right) \leq \widetilde{m}_{\rho,(E, F)}\left(A_{1}\right) \rho\left(A_{n}\right) \underset{n}{\rightarrow} 0$, hence $m\left(A_{n}\right) \underset{n}{\rightarrow} 0$. This proves the $\sigma$-additivity of $m$. Now, let $A \in \mathcal{T}_{\rho}$ with $\mu(A)=0$. With Property 4 . b), we have $\widetilde{m}_{\rho,(E, F)}(A)=0$, hence $m(A)=0$ (use Property 3.). This fact, together with the facts that $m$ is $\sigma$-additive and $\mathcal{T}_{\rho}$ is a $\delta$-ring imply the local absolute continuity in the enunciation.

Comment. (Connection with the standard variation and semivariation) Let us consider a non empty set $T$ and a $\sigma$-algebra $\mathcal{T} \subset \mathcal{P}(T)$. Also let $X, E, F$ be Banach spaces such that $X \hookrightarrow \mathcal{L}(E, F)$ and $m: \mathcal{T} \rightarrow X$ such that $m(\phi)=0$. Then, for any $A \subset T$, one can construct (see [7]): $\bar{m}(A)=\sup \left\{\sum_{i=1}^{n}\left\|m\left(A_{i}\right)\right\| \mid \mathcal{T} \ni A_{i} \subset A\right\}=$ the (standard) variation of $m$ on $A$ and $\widetilde{m}_{(E, F)}(A)=\sup \left\{\left\|\sum_{i=1}^{n} m\left(A_{i}\right)\left(x_{i}\right)\right\| \mid \mathcal{T} \ni A_{i} \subset\right.$ $\left.A, x_{i} \in E,\left\|x_{i}\right\| \leq 1\right\}=$ the (standard) $(E, F)$-semivariation of $m$ on $A$.

Theorem 3.9. The standard constructions from above are particular cases of the constructions exhibited in this section. 
Proof. One considers the measure space $(T, \mathcal{T}, \lambda)$, where $\lambda: \mathcal{T} \rightarrow \overline{\mathbf{R}}_{+}, \lambda(A)=$ $\operatorname{card}(A)$, if $A$ is finite and $\lambda(A)=\infty$, if $A$ is infinite. Also, one considers the function norm $\rho: M_{+}(\lambda) \rightarrow \overline{\mathbf{R}}_{+}$, given via $\rho(u)=\sup \{u(t) \mid t \in T\}$ for any $u \in M_{+}(\lambda)$ (actually, $\rho=\|\|_{\infty}$ for this $\lambda$ ). Then $\mathcal{T}_{\rho}=\mathcal{T}$ and one has, for any $A \subset T: \bar{m}(A)=$ $\bar{m}_{\rho}(A)$ and $\widetilde{m}_{(E, F)}(A)=\widetilde{m}_{\rho,(E, F)}(A)$.

\section{Linear and continuous operators on $\mathcal{L}_{\rho}(E)$. Integral representations}

Throughout this section, we shall consider a $\sigma$-finite and complete measure space $(T, \mathcal{T}, \mu)$, a function norm $\rho: M_{+}(\mu) \rightarrow \overline{\mathbf{R}}_{+}$and an additive measure $m: \mathcal{T}_{\rho} \rightarrow X \hookrightarrow$ $\mathcal{L}(E, F)$, where $X, E, F$ are Banach spaces.

1. For any $f=\sum_{i=1}^{n} \varphi_{A_{i}} x_{i} \in S_{E}(\rho)$, we define the elementary integral of $f$ with respect to $m$ via $\int f d m \stackrel{\text { def }}{=} \sum_{i=1}^{n} m\left(A_{i}\right)\left(x_{i}\right) \in F$. One can see that the definition of the elementary integral $\int f d m$ is coherent, i.e. it does not depend upon the representation of $f$.

We can define the elementary operator $U: S_{E}(\rho) \rightarrow F$, via $U(f)=\int f d m$.

Clearly $U$ is linear and there is a bijection between the additive measures $m: \mathcal{T}_{\rho} \rightarrow$ $X \hookrightarrow \mathcal{L}(E, F)$ and the linear operators $U: S_{E}(\rho) \rightarrow F$, given via $m \longmapsto U$, where $U(f) \stackrel{\text { def }}{=} \int f d m$. The inverse of this bijection is given via $U \longmapsto m$, where, for any $A \in \mathcal{T}_{\rho}$ and any $x \in E, m(A)(x) \stackrel{\text { def }}{=} U\left(\varphi_{A} x\right)$.

A special situation is the following. We can consider, for a given Banach space $X$, the canonical embedding (isomorphism) $X \equiv \mathcal{L}(K, X)$ and an embedding $X \hookrightarrow$ $\mathcal{L}(E, F)$. Then, for any $\varphi=\sum_{i=1}^{n} \alpha_{i} \varphi_{A_{i}} \in S(\rho)$, we consider $X \equiv \mathcal{L}(K, X)$ and $\int \varphi d m=\sum_{i=1}^{n} \alpha_{i} m\left(A_{i}\right) \in \mathcal{L}(E, F)$. This fact implies that, for any $x \in E$ and any $\varphi \in S(\rho)$, one has $\left(\int \varphi d m\right)(x)=\int \varphi x d m$.

2. Let us rewrite the definitions. Namely, one has, for any $A \subset T$ :

$$
\widetilde{m}_{\rho,(E, F)}(A)=\sup \left\{\left\|\int f d m\right\| \mid f \in S_{E}(\rho, A, 1)\right\} .
$$

Theorem 4.1. Let $A \subset T$ and $f \in S_{E}(\rho, A)$ (respectively $\varphi \in S(\rho, A)$ ). Assume that either $\rho|f| \neq 0$, or $\widetilde{m}_{\rho,(E, F)}(A)<\infty$ (respectively either $\rho|\varphi| \neq 0$, or $\left.\widetilde{m}_{\rho,(E, F)}(A)<\infty\right)$. Then we have $\left\|\int f d m\right\| \leq \widetilde{m}_{\rho,(E, F)}(A) \rho|f|$ (respectively $\left.\left\|\int \varphi d m\right\|_{o} \leq \widetilde{m}_{\rho,(K, X)}(A) \rho|\varphi| \leq \widetilde{m}_{\rho,(E, F)}(A) \rho|\varphi|\right)$.

Proof. It follows from Proposition 3.6 and Theorem 3.5.

Theorem 4.2. Assume that $\widetilde{m}_{\rho,(E, F)}(T)<\infty$.

1. If $\left(f_{n}\right)_{n}$ is a Cauchy sequence in $S_{E}(\rho) \subset \mathcal{L}_{\rho}(E)$ (respectively in $S(\rho) \subset \mathcal{L}_{\rho}$ ), then $\left(\int f_{n} d m\right)_{n}$ is a convergent sequence in $F$ (respectively in $\mathcal{L}(E, F)$ ).

2. Assume that $\left(f_{n}\right)_{n}$ and $\left(g_{n}\right)_{n}$ are sequences in $S_{E}(\rho)$ (respectively in $S(\rho)$ ) with the property that there exists $f \in \mathcal{L}_{\rho}(E)$ (respectively $f \in \mathcal{L}_{\rho}$ ) such that $f_{n} \rightarrow f$ and $g_{n} \underset{n}{\rightarrow} f$ in $\mathcal{L}_{\rho}(E)$ (respectively in $\mathcal{L}_{\rho}$ ). Then $\lim _{n} \int f_{n} d m=\lim _{n} \int g_{n} d m$.

Proof. 1. Using Theorem 4.1, we get, for any $i, j,\left\|\int f_{i} d m-\int f_{j} d m\right\|=$ $\left\|\int\left(f_{i}-f_{j}\right) d m\right\| \leq \widetilde{m}_{\rho,(E, F)}(T) \rho\left|f_{i}-f_{j}\right|$ (respectively $\left\|\int f_{i} d m-\int f_{j} d m\right\|_{o}=\| \int\left(f_{i}-\right.$ $\left.\left.f_{j}\right) d m \|_{o} \leq \widetilde{m}_{\rho,(E, F)}(T) \rho\left|f_{i}-f_{j}\right|\right)$ and this shows that the sequence $\left(\int f_{i} d m\right)_{i}$ is Cauchy, i.e. convergent. 
2. $\left\|\int f_{n} d m-\int g_{n} d m\right\|=\left\|\int\left(f_{n}-g_{n}\right) d m\right\| \leq \widetilde{m}_{\rho,(E, F)}(T) \rho\left|f_{n}-g_{n}\right| \underset{n}{\rightarrow} 0$ (respectively $\left.\left\|\int f_{n} d m-\int g_{n} d m\right\|_{o}=\left\|\int\left(f_{n}-g_{n}\right) d m\right\|_{o} \leq \widetilde{m}_{\rho,(E, F)}(T) \rho\left|f_{n}-g_{n}\right| \underset{n}{\rightarrow} 0\right)$.

Fundamental assumption. From now on, in the remainder of the paper, we shall assume that $\rho$ is of absolutely continuous type. This assumption implies (see Preliminary facts, Density Lemma) that $S_{E}(\rho)$ is dense in $\mathcal{L}_{\rho}(E)$. Hence, for an arbitrary $f \in \mathcal{L}_{\rho}(E)$, there exists a sequence $\left(f_{n}\right)_{n} \subset S_{E}(\rho)$ such that $f_{n} \underset{n}{\rightarrow} f$ in $\mathcal{L}_{\rho}(E)$. Then, Theorem 4.2 says that the sequence $\left(\int f_{n} d m\right)_{n}$ is Cauchy (in $F$ or in $\mathcal{L}(E, F)$ ) and the $\operatorname{limit}_{n} \lim _{n} \int f_{n} d m$ does not depend upon the sequence $\left(f_{n}\right)_{n}$ (it depends only upon $f$ ). We arrive at the natural

Definition 4.3. Assume that $\widetilde{m}_{\rho,(E, F)}(T)<\infty$. For any $f \in \mathcal{L}_{\rho}(E)$ (respectively $\left.f \in \mathcal{L}_{\rho}\right)$, the integral of $f$ with respect to $m$ is the element $\int f d m \stackrel{\text { def }}{=} \lim _{n} \int f_{n} d m \in F$ (respectively $\int f d m=\lim _{n} \int f_{n} d m \in \mathcal{L}(E, F)$ ), where $\left(f_{n}\right)_{n} \subset S_{E}(\rho)$ (respectively $\left.\left(f_{n}\right)_{n} \subset S(\rho)\right)$ is a sequence with the property that $f_{n} \underset{n}{\rightarrow} f$ in $\mathcal{L}_{\rho}(E)$ (respectively in $\left.\mathcal{L}_{\rho}\right)$.

Remark. The integral just defined extends the elementary integral.

Previous facts lead to

Theorem 4.4. Assume that $\widetilde{m}_{\rho,(E, F)}(T)<\infty$. We have the linear and continuous operators $U: \mathcal{L}_{\rho}(E) \rightarrow F$, given via $U(f)=\int f d m$ and $V: \mathcal{L}_{\rho} \rightarrow \mathcal{L}(E, F)$, given via $V(\varphi)=\int \varphi d m$. Then:

1. For any $\varphi \in \mathcal{L}_{\rho}$ and any $x \in E$, one has $V(\varphi)(x)=U(\varphi x)$.

2. a) $\|U\|_{o}=\widetilde{m}_{\rho,(E, F)}(T)$; b) $\|V\|_{o}=\widetilde{m}_{\rho,(K, X)}(T) \leq \widetilde{m}_{\rho,(E, F)}(T)$.

Proof. 1. Let $\varphi \in \mathcal{L}_{\rho}$ and $\left(\varphi_{n}\right)_{n} \subset \mathcal{L}_{\rho}$ such that $\varphi_{n} \underset{n}{\rightarrow} \varphi$ in $\mathcal{L}_{\rho}$. Then $\varphi_{n} x \underset{n}{\rightarrow} \varphi x$ in $\mathcal{L}_{\rho}(E)$. Hence, for any $n$, one has $V\left(\varphi_{n}\right)(x)=U\left(\varphi_{n} x\right)$ and $V(\varphi)(x)=$ $\lim _{n} V\left(\varphi_{n}\right)(x)=\lim _{n} U\left(\varphi_{n} x\right)=U(\varphi x)$.

We used the continuity of $U$ and $V$, which results from Theorem 4.1.

2. For $U$ one has $\|U\|_{o} \leq \widetilde{m}_{\rho,(E, F)}(T)$ with Theorem 4.1. The equality in the enunciation follows from equality (4.2): $\|U\|_{o}=\sup \left\{\|U(f)\|\left|f \in \mathcal{L}_{\rho}(E), \rho\right| f \mid \leq\right.$ $1\} \geq \sup \left\{\|U(f)\|\left|f \in S_{E}(\rho), \rho\right| f \mid \leq 1\right\}=\widetilde{m}_{\rho,(E, F)}(T)$.

As concerns $V$, we remark again its continuity. Using this fact, the density of $S(\rho)$ in $\mathcal{L}_{\rho}$ (because $\rho$ is of absolutely continuous type) and equality (4.2), we have: $\|V\|_{o}=\sup \left\{\|V(\varphi)\|_{o}\left|\varphi \in \mathcal{L}_{\rho}, \rho\right| \varphi \mid \leq 1\right\}=\sup \left\{\|V(\varphi)\|_{o} \mid \varphi \in S(\rho)\right.$, $\rho|\varphi| \leq 1\}=\widetilde{m}_{\rho,(K, X)}(T)$. Finally, we use Theorem 3.5.

Remark. The measure $m$ occuring in Definition 4.3 and Theorem 4.4 is $\sigma$ additive and locally absolutely continuous with respect to $\mu$ (Theorem 3.8).

The results in this subsection show that, starting with the integral, one obtains linear and continuous operators.

3. In this subsection we do the converse study, starting with linear and continuous operators and representing them as integrals. We start by considering a linear, not necessarily continuous operator $U: \mathcal{L}_{\rho}(E) \rightarrow F$. Let us define the elements $\|U\|_{\rho}$ and | $\|U \mid\|_{\rho}$ of $\overline{\mathbf{R}}_{+}$as follows:

$$
\|U\|_{\rho}=\sup \left\{\left\|\sum_{i=1}^{n} U\left(\varphi_{A_{i}} x_{i}\right)\right\| \mid \sum_{i=1}^{n} \varphi_{A_{i}} x_{i} \in S_{E}(\rho, 1)\right\},
$$




$$
\|U \mid\|_{\rho}=\sup \left\{\sum_{i=1}^{n}\left\|U\left(\varphi_{A_{i}} x_{i}\right)\right\| \mid \sum_{i=1}^{n} \varphi_{A_{i}} x_{i} \in S_{E}(\rho, 1)\right\} .
$$

Clearly, one has the following properties:

$0 \leq\|U\|_{\rho} \leq\left|\left\|U\left|\left\|_{\rho} \leq \infty ;\right\| U+V\left\|_{\rho} \leq\right\| U\left\|_{\rho}+\right\| V\left\|_{\rho} ;\left|\left\|U+V\left|\left\|_{\rho} \leq\right\|\|U \mid\|_{\rho}+\right.\right.\right.\right.\right.\right.\right.$ $\left|\left\|V\left|\left\|_{\rho} ;\right\| \alpha U\left\|_{\rho}=|\alpha|\right\| U\left\|_{\rho} ;\left|\left\|\alpha U\left|\left\|_{\rho}=|\alpha|\left|\|U \mid\|_{\rho}\right.\right.\right.\right.\right.\right.\right.\right.\right.$ (for any linear $U, V: \mathcal{L}_{\rho}(E) \rightarrow F$ and for any $\alpha \in K$, with the convention $0 \cdot \infty=0$ ).

Proposition 4.5. Let $f=\sum_{i=1}^{k} \varphi_{A_{i}} x_{i} \in S_{E}(\rho, 1)$. Assuming that either $\|U\|_{\rho}<$ $\infty$, or $\rho|f| \neq 0$ (respectively either $\left|\|U \mid\|_{\rho}<\infty\right.$, or $\left.\rho\right| f \mid \neq 0$ ), one has $\|U(f)\|=$ $\left\|\sum_{i=1}^{k} U\left(\varphi_{A_{i}} x_{i}\right)\right\| \leq\|U\|_{\rho} \rho|f|$ (respectively $\sum_{i=1}^{k}\left\|U\left(\varphi_{A_{i}} x_{i}\right)\right\| \leq\left|\left\|U\left|\|_{\rho} \rho\right| f \mid\right)\right.$.

Proof. First, we work for $\|U\|_{\rho}$. Assume that either $\|U\|_{\rho}<\infty$, or $\rho|f| \neq 0$. The case $\|U\|_{\rho}=\infty$ (hence $\rho|f|>0$ ) is trivial, so let us assume that $\|U\|_{\rho}<\infty$. If $\rho|f| \neq 0$, we have $\frac{1}{\rho|f|} f \in S_{E}(\rho, 1)$, hence $\left\|U\left(\frac{1}{\rho|f|} f\right)\right\| \leq\|U\|_{\rho}$. Now, assume that $\rho|f|=0$ and prove that $U(f)=0$. Indeed, if $U(f) \neq 0$, because $\rho|n f|=0$, we have $n\|U(f)\|=\|U(n f)\| \leq\|U\|_{\rho}$ for any $n \in \mathbf{N}$, leading to $\|U\|_{\rho}=\infty$, absurd.

What concerns ||$|U| \|_{\rho}$, we work on the non trivial case $\left|\|U \mid\|_{\rho}<\infty\right.$. If $\left.\rho\right| f \mid \neq$ 0 , use again $\frac{1}{\rho|f|} f=\sum_{i=1}^{k} \varphi_{A_{i}} \frac{1}{\rho|f|} x_{i} \in S_{E}(\rho, 1)$ to obtain $\sum_{i=1}^{k}\left\|U\left(\varphi_{A_{i}} \frac{1}{\rho|f|} x_{i}\right)\right\|=$ $\frac{1}{\rho|f|} \sum_{i=1}^{k}\left\|U\left(\varphi_{A_{i}} x_{i}\right)\right\| \leq\left|\|U \mid\|_{\rho}\right.$. Finally, if $\left.\rho\right| f \mid=0$, we have $n f=\sum_{i=1}^{k} \varphi_{A_{i}} n x_{i} \in$ $S_{E}(\rho, 1)$, hence $\sum_{i=1}^{k}\left\|U\left(\varphi_{A_{i}} n x_{i}\right)\right\|=n \sum_{i=1}^{k}\left\|U\left(\varphi_{A_{i}} x_{i}\right)\right\| \leq\|U \mid\|_{\rho}$ for any $n \in \mathbf{N}$. This implies $\sum_{i=1}^{k}\left\|U\left(\varphi_{A_{i}} x_{i}\right)\right\|=0$ a.s.o.

Sometimes, the inequality $\|U\|_{\rho} \leq\left|\|U \mid\|_{\rho}\right.$ is actually an equality, as in

Theorem 4.6. If $F=K$ or if $\rho=\|\|_{1}$, we have $\|U\|_{\rho}=\|\| U \|_{\rho}$.

Proof. 1. Assume that $F=K$ (hence $\left.U \in\left(\mathcal{L}_{\rho}(E)\right)^{\prime}\right)$. We must prove that $\left|\|U \mid\|_{\rho} \leq\|U\|_{\rho}\right.$. Take an arbitrary $f=\sum_{i=1}^{n} \varphi_{A_{i}} x_{i} \in S_{E}(\rho, 1)$. For any $i=1,2, \ldots, n$ find $\theta_{i} \in K$ with $\left|\theta_{i}\right|=1$ such that $\left|U\left(\varphi_{A_{i}} x_{i}\right)\right|=\theta_{i} U\left(\varphi_{A_{i}} x_{i}\right)=U\left(\varphi_{A_{i}} \theta_{i} x_{i}\right)$. Clearly $g=\sum_{i=1}^{n} \varphi_{A_{i}} \theta_{i} x_{i} \in S_{E}(\rho, 1)$, hence $\left|\sum_{i=1}^{n} U\left(\varphi_{A_{i}} \theta_{i} x_{i}\right)\right| \leq\|U\|_{\rho}$. But $\sum_{i=1}^{n}\left|U\left(\varphi_{A_{i}} x_{i}\right)\right|$ $=\sum_{i=1}^{n} U\left(\varphi_{A_{i}} \theta_{i} x_{i}\right)=\left|\sum_{i=1}^{n} U\left(\varphi_{A_{i}} \theta_{i} x_{i}\right)\right| \leq\|U\|_{\rho}$. Because $f$ is arbitrary, we can pass to supremum with respect to $f$ and get $\left|\|U \mid\|_{\rho} \leq\|U\|_{\rho}\right.$.

2. Now, assume that $\rho=\|\|_{1}$. We work in the non trivial case $\|U\|_{\rho}<\infty$. Take an arbitrary $f=\sum_{i=1}^{n} \varphi_{A_{i}} x_{i} \in S_{E}(\rho, 1)$. Then $\rho|f|=\|f\|_{1}=\sum_{i=1}^{n} \mu\left(A_{i}\right)\left\|x_{i}\right\|=$ $\sum_{i=1}^{n}\left\|\varphi_{A_{i}} x_{i}\right\|_{1}$. Because $\|U\|_{\rho}<\infty$, we can apply Proposition 4.5. We get

$$
\sum_{i=1}^{n}\left\|U\left(\varphi_{A_{i}} x_{i}\right)\right\| \leq \sum_{i=1}^{n}\|U\|_{\rho} \rho\left|\varphi_{A_{i}} x_{i}\right|=\|U\|_{\rho} \sum_{i=1}^{n}\left\|\varphi_{A_{i}} x_{i}\right\|_{1}=\|U\|_{\rho} \rho|f| \leq\|U\|_{\rho} .
$$

Passing to supremum with respect to arbitrary $f$, we get $\left|\|U \mid\|_{\rho} \leq\|U\|_{\rho}\right.$.

From now on, we shall deal in this subsection with linear and continuous operators $U: \mathcal{L}_{\rho}(E) \rightarrow F$. For such an operator $U$, we have

$$
\|U\|_{\rho}=\|U\|_{o} .
$$

Indeed, because $S_{E}(\rho)$ is dense in $\mathcal{L}_{\rho}(E)$ (due to the fact that $\rho$ is of absolutely continuous type), it follows that $S_{E}(\rho, 1)$ is dense in $\left\{f \in \mathcal{L}_{\rho}(E)|\rho| f \mid \leq 1\right\}$. Hence, 
due to the continuity of $U$, we have $\|U\|_{o}=\sup \left\{\|U(f)\|\left|f \in \mathcal{L}_{\rho}(E), \rho\right| f \mid \leq 1\right\}=$ $\sup \left\{\|U(f)\| \mid f \in S_{E}(\rho, 1)\right\}=\|U\|_{\rho}$.

The following fundamental result makes more precise Theorem 4.4 and expresses the main idea (integral representation) of this paragraph. We shall deal directly with measures $m: \mathcal{T}_{\rho} \rightarrow \mathcal{L}(E, F)$, omitting the explicit reference to the embedding $X \hookrightarrow \mathcal{L}(E, F)$.

Theorem 4.7. (Integral representation of linear and continuous operators on $\left.\mathcal{L}_{\rho}(E)\right)$ There exists a linear isomorphism $U \longleftrightarrow m$ between the vector space of all linear and continuous operators $U: \mathcal{L}_{\rho}(E) \rightarrow F$ and the vector space of all $\sigma$-additive measures $m: \mathcal{T}_{\rho} \rightarrow \mathcal{L}(E, F)$ with $\widetilde{m}_{\rho,(E, F)}(T)<\infty$ which are locally absolutely continuous with respect to $\mu$. This isomorphism is given as follows: If $U$ and $m$ are in the correspondence described above, then

$$
U(f)=\int f d m
$$

for any $f \in \mathcal{L}_{\rho}(E)$. Supplementarily, we have $\|U\|_{\rho}=\|U\|_{o}=\widetilde{m}_{\rho,(E, F)}(T)$ and $\|U \mid\|_{\rho}=\bar{m}_{\rho}(T)$.

Proof. A. Theorem 4.4 furnishes the first part of the proof. Namely, starting with a measure $m$ like in the enunciation, we obtain the linear and continuous operator $U: \mathcal{L}_{\rho}(E) \rightarrow F$ acting as in (4.4). As we have seen (equality (4.3)), we have $\|U\|_{o}=\|U\|_{\rho}$. But $\|U\|_{\rho}=\sup \left\{\left\|\sum_{i=1}^{n} U\left(\varphi_{A_{i}} x_{i}\right)\right\| \mid \sum_{i=1}^{n} \varphi_{A_{i}} x_{i} \in S_{E}(\rho, 1)\right\}$. Because $U\left(\varphi_{A_{i}} x_{i}\right)=\int \varphi_{A_{i}} x_{i} d m=m\left(A_{i}\right)\left(x_{i}\right)$ it follows that $\|U\|_{\rho}=\sup \left\{\left\|\sum_{i=1}^{n} m\left(A_{i}\right)\left(x_{i}\right)\right\| \mid\right.$ $\left.\sum_{i=1}^{n} \varphi_{A_{i}} x_{i} \in S_{E}(\rho, 1)\right\}=\widetilde{m}_{\rho,(E, F)}(T)$. The equality $\|U \mid\|_{\rho}=\bar{m}_{\rho}(T)$ is obtained likewise.

B. Conversely, take an operator $U: \mathcal{L}_{\rho}(E) \rightarrow F$ which is linear and continuous. Hence $\|U\|_{o}=\|U\|_{\rho}<\infty$. Using $U$ we define $m: \mathcal{T}_{\rho} \rightarrow \mathcal{L}(E, F), m(A)(x) \stackrel{\text { def }}{=} U\left(\varphi_{A} x\right)$ for any $A \in \mathcal{T}_{\rho}$ and any $x \in E$. The linearity and the continuity of all $m(A): E \rightarrow F$ follow from the linearity and the continuity of $U$ as well as the additivity of $m$. For any $f=\sum_{i=1}^{n} \varphi_{A_{i}} x_{i} \in S_{E}(\rho, 1)$, one has: $\left\|\sum_{i=1}^{n} m\left(A_{i}\right)\left(x_{i}\right)\right\|=\left\|\sum_{i=1}^{n} U\left(\varphi_{A_{i}} x_{i}\right)\right\|=$ $\|U(f)\|$. Passing to supremum with respect to $f \in S_{E}(\rho, 1)$, we obtain the equality $\widetilde{m}_{\rho,(E, F)}(T)=\|U\|_{o}$, hence $\widetilde{m}_{\rho,(E, F)}(T)<\infty$. Theorem 3.8 asserts that $m$ is $\sigma$ additive and locally absolutely continuous with respect to $\mu$. In the same way, we obtain the equality $\bar{m}_{\rho}(T)=\left|\|U \mid\|_{\rho}\right.$.

C. The final part of the proof consists in proving that the correspondences exhibited at points $\mathrm{A}$ and $\mathrm{B}$, i.e. $m \longmapsto U$ and $U \longmapsto m$ are mutually inverse.

a) Start with the measure $m: \mathcal{T}_{\rho} \rightarrow \mathcal{L}(E, F)$ as in the enunciation. Construct the operator $U: \mathcal{L}_{\rho}(E) \rightarrow F$, via $U(f)=\int f d m$ for any $f \in \mathcal{L}_{\rho}(E)$. Then define the measure $n: \mathcal{T}_{\rho} \rightarrow \mathcal{L}(E, F)$, via $n(A)(x)=U\left(\varphi_{A} x\right)$ for any $A \in \mathcal{T}_{\rho}$ and any $x \in E$. We see that $n(A)(x)=\int \varphi_{A} x d m=m(A)(x)$, hence $n=m$.

b) Start with the linear and continuous operator $U: \mathcal{L}_{\rho}(E) \rightarrow F$. Construct the measure $m: \mathcal{T}_{\rho} \rightarrow \mathcal{L}(E, F)$, given via $m(A)(x)=U\left(\varphi_{A} x\right)$ for any $A \in \mathcal{T}_{\rho}$ and any $x \in E$. Now, define the linear and continuous operator $V: \mathcal{L}_{\rho}(E) \rightarrow F$, via $V(f)=\int f d m$ for any $f \in \mathcal{L}_{\rho}(E)$. For any $A \in \mathcal{T}_{\rho}$ and any $x \in E, V\left(\varphi_{A} x\right)=$ $\int \varphi_{A} x d m=m(A)(x)=U\left(\varphi_{A} x\right)$. Hence $V(f)=U(f)$ for any $f \in S_{E}(\rho)$. Because $S_{E}(\rho)$ is dense in $\mathcal{L}_{\rho}(E)$ and $V, U$ are continuous, it follows that $V=U$.

Corollary 4.8. There exists a linear and isometric isomorphism $U \longleftrightarrow m$ between the Banach space $\mathcal{L} \mathcal{L}\left(\mathcal{L}_{\rho}(E), F\right)$ of all linear and continuous operators 
$U: \mathcal{L}_{\rho}(E) \rightarrow F$ with the property that $\|U\|_{\rho}<\infty$, equipped with the norm $U \longmapsto\left|\|U \mid\|_{\rho}\right.$ and the Banach space $\operatorname{cabv}\left(\mathcal{T}_{\rho},(E, F), \mu\right)$ of all $\sigma$-additive measures $m: \mathcal{T}_{\rho} \rightarrow \mathcal{L}(E, F)$ which are locally absolutely continuous with respect to $\mu$, with the property that $\bar{m}_{\rho}(T)<\infty$, equipped with the norm $m \longmapsto \bar{m}_{\rho}(T)$. The correspondence $U \longleftrightarrow m$ is that one given by Theorem 4.7.

Proof. The linear isomorphism in the enunciation is clear. On $\mathcal{L} \mathcal{L}\left(\mathcal{L}_{\rho}(E), F\right)$ one has the norm $U \longmapsto \mid\|U\|_{\rho} \geq\|U\|_{\rho}=\|U\|_{o}$ and this leads to the fact that on $\operatorname{cabv}\left(\mathcal{T}_{\rho},(E, F), \mu\right)$ one has the corresponding norm $m \longmapsto \bar{m}_{\rho}(T)=\left|\|U \mid\|_{\rho}\right.$ (if $U \longleftrightarrow m)$. So, the isomorphism is an isometry too.

It remains to be proved that $\mathcal{L} \mathcal{L}\left(\mathcal{L}_{\rho}(E), F\right)$ is a Banach space for the norm $U \longmapsto\left|\|U \mid\|_{\rho}\right.$. To this end, take a Cauchy sequence $\left(U_{n}\right)_{n}$ in this space. Because $\left|\|U \mid\|_{\rho} \geq\|U\|_{\rho}\right.$, it follows that $\left(U_{n}\right)_{n}$ is Cauchy in the Banach space $\mathcal{L}\left(\mathcal{L}_{\rho}(E), F\right)$ (equipped with the norm $\left.U \longmapsto\|U\|_{o}=\|U\|_{\rho}\right)$. Let $U \in \mathcal{L}_{\rho}(E)$ be the limit (in $\left.\mathcal{L}\left(\mathcal{L}_{\rho}(E), F\right)\right)$ of $\left(U_{n}\right)_{n}$. First, we show that $U \in \mathcal{L} \mathcal{L}\left(\mathcal{L}_{\rho}(E), F\right)$. Indeed, the sequence $\left(U_{n}\right)_{n}$ is bounded in $\mathcal{L} \mathcal{L}\left(\mathcal{L}_{\rho}(E), F\right)$, being Cauchy. Let $M>0$ be a number such that $\left|\left\|U_{n} \mid\right\|_{\rho}<M\right.$ for any $n$. Take an arbitrary $f=\sum_{i=1}^{k} \varphi_{A_{i}} x_{i} \in S_{E}(\rho, 1)$. Then, for any $n \in \mathbf{N}$, one has $\sum_{i=1}^{k}\left\|U_{n}\left(\varphi_{A_{i}} x_{i}\right)\right\| \leq\left|\left\|U_{n} \mid\right\|_{\rho}<M\right.$. Because $\left\|U_{n}-U\right\|_{\rho} \rightarrow 0$, it follows that $U_{n} \underset{n}{\rightarrow} U$ pointwise, hence $U_{n}\left(\varphi_{A_{i}} x_{i}\right) \underset{n}{\rightarrow} U\left(\varphi_{A_{i}} x_{i}\right)$ for any $i$. This leads to $\sum_{i=1}^{k}\left\|U\left(\varphi_{A_{i}} x_{i}\right)\right\| \leq M$ and $f$ being arbitrary, we have $\|U\| \|_{\rho} \leq M<\infty$, i.e. $U \in \mathcal{L} \mathcal{L}\left(\mathcal{L}_{\rho}(E), F\right)$.

The final step consists in proving that $\left|\left\|U_{n}-U \mid\right\|_{\rho} \rightarrow 0\right.$. Indeed, take an arbitrary $\varepsilon>0$ and an arbitrary $f=\sum_{i=1}^{k} \varphi_{A_{i}} x_{i} \in S_{E}(\rho, 1)$. We consider $p_{0} \in \mathbf{N}$ such that, if $p \geq p_{0}$ and $q \geq p_{0}$, one has $\left\|U_{p}-U_{q} \mid\right\|_{\rho}<\frac{\varepsilon}{2}$. Then $\sum_{i=1}^{k}\left\|U_{p}\left(\varphi_{A_{i}} x_{i}\right)-U_{q}\left(\varphi_{A_{i}} x_{i}\right)\right\| \leq$ \|\|$U_{p}-U_{q} \mid \|_{\rho}<\frac{\varepsilon}{2}$. Passing to $q$-limit, we get $\sum_{i=1}^{k}\left\|U_{p}\left(\varphi_{A_{i}} x_{i}\right)-U\left(\varphi_{A_{i}} x_{i}\right)\right\|<\frac{\varepsilon}{2}$. Because $f$ is arbitrary, this leads to $\left\|U_{p}-U \mid\right\|_{\rho} \leq \frac{\varepsilon}{2}<\varepsilon$ a.s.o.

The following example illustrates the concrete application of the theory.

Example 4.9. We refer to the construction of the operator $V$ in Theorem 4.4. Namely, we take $\rho=\|\|_{p}, 1 \leq p<\infty$, hence $\mathcal{L}_{\rho}=\mathcal{L}^{p}(\mu)$. Also, we take $X=$ $L^{p}(\mu), E=K, F=X$, hence $X \equiv \mathcal{L}(K, X)$. The measure $m: \mathcal{T}_{\rho} \rightarrow L^{p}(\mu)$ is given via $m(A)=\widetilde{\varphi}_{A}$. For the non trivial case $\mu \not \equiv 0$, we have $\widetilde{m}_{\rho,(K, X)}(T)=$ $\sup \left\{\left\|\sum_{i=1}^{n} \alpha_{i} \widetilde{\varphi}_{A_{i}}\right\|_{p} \mid \sum_{i=1}^{n} \alpha_{i} \varphi_{A_{i}} \in S(\rho, 1)\right\}=1$ and we can construct $V: \mathcal{L}_{\rho} \rightarrow$ $L_{\rho}=L^{p}(\mu)=X$, via $V(\varphi)=\int \varphi d m$. For any $\varphi=\sum_{i=1}^{n} \alpha_{i} \varphi_{A_{i}} \in S(\rho)$, we have $V(\varphi)=\int \varphi d m=\sum_{i=1}^{n} \alpha_{i} \widetilde{\varphi}_{A_{i}}=\widetilde{\varphi}$. Due to the continuity of $V$ and to the fact that $S(\rho)$ is dense in $\mathcal{L}_{\rho}$, it follows that $V(\varphi)=\widetilde{\varphi}$ for any $\varphi \in \mathcal{L}_{\rho}$. Also, it follows that the associated operator $\widetilde{V}: L_{\rho} \rightarrow L_{\rho}$ acts via $\widetilde{V}(\widetilde{\varphi})=V(\varphi)=\widetilde{\varphi}$, hence $\widetilde{V}$ is the identity operator of $L_{\rho}$.

\section{Natural operators}

Again, in this section, we shall consider a $\sigma$-finite and complete measure space $(T, \mathcal{T}, \mu)$, a function norm $\rho: M_{+}(\mu) \rightarrow \overline{\mathbf{R}}_{+}$and an additive measure $m: \mathcal{T}_{\rho} \rightarrow X \hookrightarrow$ $\mathcal{L}(E, F)$, where $X, E, F$ are Banach spaces. We add the fact that $\rho$ will be assumed to be of absolutely continuous type. The basic result of this section is

Theorem 5.1. There exists a linear, injective and continuous map $\Omega: \mathcal{L}\left(\mathcal{L}_{\rho}(E), F\right)$ $\rightarrow \mathcal{L}\left(\mathcal{L}_{\rho}, \mathcal{L}(E, F)\right)$ acting via $\Omega(U)=U^{*}$ for any $U \in \mathcal{L}\left(\mathcal{L}_{\rho}(E), F\right)$, where $U^{*}$ is defi- 
ned as follows: for any $\varphi \in \mathcal{L}_{\rho}$ and any $x \in E, U^{*}(\varphi)(x)=U(\varphi x)$. We have $\left\|U^{*}\right\|_{o}=\left\|U^{*}\right\|_{\rho} \leq\|U\|_{\rho}=\|U\|_{o}$, hence $\|\Omega\|_{o} \leq 1$.

Proof. The definition in the enunciation is "good". Indeed, for any $\varphi \in \mathcal{L}_{\rho}$ and any $x \in E$, one has $\|U(\varphi x)\| \leq\|U\|_{o} \rho|\varphi|\|x\|$. This shows that: $\left.a\right)$ The linear map $x \longmapsto U(\varphi x)$ is continuous; call it $U^{*}(\varphi): E \rightarrow F$ and we have $\left\|U^{*}(\varphi)\right\|_{o} \leq\|U\|_{o} \rho|\varphi|$; b) The linear map $\varphi \longmapsto U^{*}(\varphi)$ is continuous; call it $U^{*}: \mathcal{L}_{\rho} \rightarrow \mathcal{L}(E, F)$ and we have $\left\|U^{*}\right\|_{o} \leq\|U\|_{o}$. Hence $\left\|U^{*}\right\|_{\rho}=\left\|U^{*}\right\|_{o} \leq\|U\|_{o}=\|U\|_{\rho}$. Clearly we defined the linear map $\Omega: \mathcal{L}\left(\mathcal{L}_{\rho}(E), F\right) \rightarrow \mathcal{L}\left(\mathcal{L}_{\rho}, \mathcal{L}(E, F)\right)$ via $\Omega(U)=U^{*}$ and $\|\Omega(U)\|_{o} \leq\|U\|_{o}$, hence $\Omega$ is continuous with $\|\Omega\|_{o} \leq 1$. Finally, we prove that $\Omega$ is injective, showing that for an arbitrary $U \in \operatorname{Ker}(\Omega)$, one has $U=0$. Indeed, for such $U$ one has $U^{*}=0$, hence $U^{*}(\varphi)=0$ for any $\varphi \in \mathcal{L}_{\rho}$, i.e. $U^{*}(\varphi)(x)=0$ for any $\varphi \in \mathcal{L}_{\rho}$ and any $x \in E$. Take an arbitrary $f=\sum_{i=1}^{n} \varphi_{A_{i}} x_{i} \in S_{E}(\rho)$. Then $U(f)=\sum_{i=1}^{n} U\left(\varphi_{A_{i}} x_{i}\right)=$ $\sum_{i=1}^{n} U^{*}\left(\varphi_{A_{i}}\right)\left(x_{i}\right)=0$. We proved that the continuous map $U$ vanishes on the set $S_{E}(\rho)$ which is dense in $\mathcal{L}_{\rho}(E)$, hence one must have $U=0$.

The operators $U$ and $U^{*}$ described in the preceding result are generated by the same measure, as it is shown in

Theorem 5.2. Use the same notations in Theorem 5.1. For $U \in \mathcal{L}\left(\mathcal{L}_{\rho}(E), F\right)$, let $m: \mathcal{T}_{\rho} \rightarrow \mathcal{L}(E, F)$ be the $\sigma$-additive measure defining $U$ (Theorem 4.7), i.e. $U(f)=\int f d m$ for any $f \in \mathcal{L}_{\rho}(E)$ and $\widetilde{m}_{\rho,(E, F)}(T)<\infty$. Then $m$ defines $U^{*}$ too, i.e. $U^{*}(\varphi)=\int \varphi d m$ for any $\varphi \in \mathcal{L}_{\rho}$. We also have $\left\|U^{*}\right\|_{\rho} \leq\|U\|_{\rho}$ and $\left|\left\|U^{*}\left|\left\|_{\rho}=\left|\|U \mid\|_{\rho}\right.\right.\right.\right.\right.$.

Proof. According to Theorem 4.4 we can define the linear and continuous operator $U_{1}: \mathcal{L}_{\rho} \rightarrow \mathcal{L}(E, F)$ via $U_{1}(\varphi)=\int \varphi d m$. We must prove that $U_{1}=U^{*}$, i.e. we must prove that $U_{1}(\varphi)(x)=U(\varphi x)$ for any $\varphi \in \mathcal{L}_{\rho}$ and any $x \in E$. This will be proved first for an arbitrary $\varphi=\sum_{i=1}^{n} \varphi_{A_{i}} \alpha_{i} \in S(\rho)$. Indeed, $U_{1}(\varphi)(x)=$ $\left(\int\left(\sum_{i=1}^{n} \varphi_{A_{i}} \alpha_{i}\right) d m\right)(x)=\left(\sum_{i=1}^{n} \alpha_{i} m\left(A_{i}\right)\right)(x)=\sum_{i=1}^{n} \alpha_{i} m\left(A_{i}\right)(x)$. On the other hand, $U(\varphi x)=\int\left(\sum_{i=1}^{n} \varphi_{A_{i}} \alpha_{i} x\right) d m=\sum_{i=1}^{n} m\left(A_{i}\right)\left(\alpha_{i} x\right)=\sum_{i=1}^{n} \alpha_{i} m\left(A_{i}\right)(x)$. Now, let $\varphi \in \mathcal{L}_{\rho}$ be arbitrary and let $\left(\varphi_{n}\right)_{n} \subset S(\rho)$ such that $\varphi_{n} \underset{n}{\rightarrow} \varphi$ in $\mathcal{L}_{\rho}$. Then, for any $x \in E, \varphi_{n} x \underset{n}{\rightarrow} \varphi x$ in $\mathcal{L}_{\rho}(E)$, hence $U_{1}\left(\varphi_{n}\right)(x) \underset{n}{\rightarrow} U_{1}(\varphi)(x)$ and $U\left(\varphi_{n} x\right) \underset{n}{\rightarrow} U(\varphi x)$ (continuity of $U$ and $U_{1}$ ) and these facts imply $U_{1}(\varphi)(x)=U(\varphi x)$.

As we have seen in the previous result, we have $\left\|U^{*}\right\|_{\rho} \leq\|U\|_{\rho}$ and we must prove that $\left|\left\|U^{*}\left|\left\|_{\rho}=\left|\|U \mid\|_{\rho}\right.\right.\right.\right.\right.$. To this end take $A \in \mathcal{T}_{\rho}$ and $\alpha \in K$. We have

$$
\begin{aligned}
\left\|U^{*}\left(\varphi_{A} \alpha\right)\right\|_{o} & =\sup _{\|x\| \leq 1}\left\|U^{*}\left(\varphi_{A} \alpha\right)(x)\right\|=\sup _{\|x\| \leq 1}|\alpha|\left\|U^{*}\left(\varphi_{A}\right)(x)\right\| \\
& =\sup _{\|x\| \leq 1}|\alpha|\left\|U\left(\varphi_{A} x\right)\right\|=\sup _{\|x\| \leq 1}|\alpha|\|m(A)(x)\|=|\alpha|\|m(A)\|_{o} .
\end{aligned}
$$

Consequently, if $S(\rho) \ni \varphi=\sum_{i=1}^{n} \varphi_{A_{i}} \alpha_{i}, \rho|\varphi| \leq 1$, one has $\sum_{i=1}^{n}\left\|U^{*}\left(\varphi_{A_{i}} \alpha_{i}\right)\right\|_{o}=$ $\sum_{i=1}^{n}\left|\alpha_{i}\right|\left\|m\left(A_{i}\right)\right\|_{o}$ and passing to supremum over all possible $\varphi \in S(\rho), \rho|\varphi| \leq 1$, we obtain ||$U^{*}\left|\left\|_{\rho}=\bar{m}_{\rho}(T)=\left|\|U \mid\|_{\rho}\right.\right.\right.$.

It is time to define the natural operators (special elements in the image of the embedding $\Omega$ ).

Definition 5.3. A linear and continuous operator $V: \mathcal{L}_{\rho} \rightarrow \mathcal{L}(E, F)$ will be called ( $\rho, E, F)$-natural (many times simply natural) in case there exists $U \in \mathcal{L}\left(\mathcal{L}_{\rho}(E), F\right)$ such that $V=\Omega(U)$ (i.e. one has $V \in \Omega\left(\mathcal{L}\left(\mathcal{L}_{\rho}(E), F\right)\right)$ ). We shall denote

$$
\operatorname{Nat}(\rho, E, F) \stackrel{\text { def }}{=} \Omega\left(\mathcal{L}\left(\mathcal{L}_{\rho}(E), F\right)\right)
$$


It is seen that $\mathcal{L}\left(\mathcal{L}_{\rho}(E), F\right)$ and $\operatorname{Nat}(\rho, E, F)$ are isomorphic linear spaces, the isomorphism $\Omega_{r}: \mathcal{L}\left(\mathcal{L}_{\rho}(E), F\right) \rightarrow \operatorname{Nat}(\rho, E, F)$ being given by $\Omega_{r}(U)=\Omega(U)$ for any $U \in \mathcal{L}\left(\mathcal{L}_{\rho}(E), F\right)$.

Now, we shall restrict and corestrict the isomorphism $\Omega_{r}$. Namely, let

$$
\begin{gathered}
\mathcal{L} \mathcal{L}\left(\mathcal{L}_{\rho}(E), F\right) \stackrel{\text { def }}{=}\left\{U \in \mathcal{L}\left(\mathcal{L}_{\rho}(E), F\right)\|\| U \mid \|_{\rho}<\infty\right\}, \\
\mathcal{L} \mathcal{L}\left(\mathcal{L}_{\rho}, \mathcal{L}(E, F)\right)=\left\{V \in \mathcal{L}\left(\mathcal{L}_{\rho}, \mathcal{L}(E, F)\right)\|\| V \|_{\rho}<\infty\right\} .
\end{gathered}
$$

Previous facts say that we have the linear injection $I: \mathcal{L} \mathcal{L}\left(\mathcal{L}_{\rho}(E), F\right) \rightarrow \mathcal{L} \mathcal{L}\left(\mathcal{L}_{\rho}, \mathcal{L}(E, F)\right)$, $I(U)=\Omega_{r}(U)=\Omega(U)$ for any $U \in \mathcal{L} \mathcal{L}\left(\mathcal{L}_{\rho}(E), F\right)$. Actually $I$ is a bijection.

Theorem 5.4. The linear map $I$ is a bijection (linear isomorphism). In particular, if $\rho=\|\|_{1}$, we have $\mathcal{L} \mathcal{L}\left(\mathcal{L}_{\rho}(E), F\right)=\mathcal{L}\left(\mathcal{L}_{\rho}(E), F\right), \mathcal{L} \mathcal{L}\left(\mathcal{L}_{\rho}, \mathcal{L}(E, F)\right)=$ $\mathcal{L}\left(\mathcal{L}_{\rho}, \mathcal{L}(E, F)\right)$, hence $I=\Omega$ and $\operatorname{Nat}(\rho, E, F)=\mathcal{L}\left(\mathcal{L}_{\rho}, \mathcal{L}(E, F)\right)$.

Proof. Let $U_{1} \in \mathcal{L} \mathcal{L}\left(\mathcal{L}_{\rho}, \mathcal{L}(E, F)\right)$. We shall find $U \in \mathcal{L} \mathcal{L}\left(\mathcal{L}_{\rho}(E), F\right)$ such that $U_{1}=I(U)=\Omega(U)$, thus proving that $I$ is surjective.

Writing $\mathcal{L}(E, F)=X$ we actually have $U_{1} \in \mathcal{L} \mathcal{L}\left(\mathcal{L}_{\rho}, X\right)=\mathcal{L} \mathcal{L}\left(\mathcal{L}_{\rho}(K), X\right) \subset$ $\mathcal{L}\left(\mathcal{L}_{\rho}(K), X\right)$. Because $\left|\left\|U_{1} \mid\right\|_{\rho}<\infty\right.$, we apply Corollary 4.8 and find a $\sigma$-additive measure $m: \mathcal{T}_{\rho} \rightarrow \mathcal{L}(K, X) \equiv X=\mathcal{L}(E, F)$ such that $\bar{m}_{\rho}(T)=\left|\left\|U_{1} \mid\right\|_{\rho}<\infty\right.$ and $U_{1}(\varphi)=\int \varphi d m$ for any $\varphi \in \mathcal{L}_{\rho}$. Using an isomorphic copy we can consider that $m: \mathcal{T}_{\rho} \rightarrow \mathcal{L}(E, F)$. Because $\widetilde{m}_{\rho,(E, F)}(T) \leq \bar{m}_{\rho}(T)<\infty$ we apply Theorem 4.4 to define $U \in \mathcal{L}\left(\mathcal{L}_{\rho}(E), F\right)$ such that $U(f)=\int f d m$ for any $f \in \mathcal{L}_{\rho}(E)$ and having the property $\left|\|U \mid\|_{\rho}=\bar{m}_{\rho}(T)<\infty\right.$. Hence $U \in \mathcal{L} \mathcal{L}\left(\mathcal{L}_{\rho}(E), F\right)$. We finally prove that $U_{1}=I(U)$, i.e. we prove that $U_{1}(\varphi)(x)=U(\varphi x)$ for any $\varphi \in \mathcal{L}_{\rho}$ and any $x \in E$. This is done like in the proof of Theorem 5.2.

The particular case $\rho=\|\|_{1}$ follows from Theorem 4.6.

\section{Complementary considerations concerning natural operators.}

1. Assume that $V: \mathcal{L}_{\rho} \rightarrow \mathcal{L}(E, F)$ is linear. Define the linear operator $U_{1}(V)$ : $S_{E}(\rho) \rightarrow F$ via $U_{1}(V)\left(\varphi=\sum_{i=1}^{n} \varphi_{A_{i}} x_{i}\right) \stackrel{\text { def }}{=} \sum_{i=1}^{n} V\left(\varphi_{A_{i}}\right)\left(x_{i}\right)$. The definition of $U_{1}(V)$ is coherent (not depending upon the representation of $\varphi$ ) because $U_{1}(V)(\varphi)=\int \varphi d m$, where $m: \mathcal{T}_{\rho} \rightarrow \mathcal{L}(E, F)$ is the additive measure defined by $m(A)(x)=V\left(\varphi_{A}\right)(x)$ for any $A \in \mathcal{T}_{\rho}$ and any $x \in E$.

2. Now, let $V \in \mathcal{L}\left(\mathcal{L}_{\rho}, \mathcal{L}(E, F)\right)$ and assume that $V$ is $(\rho, E, F)$-natural. Then, there exists an unique linear and continuous $U(V) \stackrel{\text { def }}{=} U: \mathcal{L}_{\rho}(E) \rightarrow F$ such that $V=\Omega(U)$, i.e. $U(\varphi x)=V(\varphi)(x)$ for any $\varphi \in \mathcal{L}_{\rho}$ and any $x \in E$. So, for any $\varphi=\sum_{i=1}^{n} \varphi_{A_{i}} x_{i} \in S_{E}(\rho)$ one has the formula for the computation of $U$ on $S_{E}(\rho)$ :

$$
U(\varphi)=\sum_{i=1}^{n} U\left(\varphi_{A_{i}} x_{i}\right)=\sum_{i=1}^{n} V\left(\varphi_{A_{i}}\right)\left(x_{i}\right)=U_{1}(V)(\varphi) .
$$

Because $U$ is continuous, there exists $0<M<\infty$ such that $\|U(\varphi)\| \leq M \rho|\varphi|$ for any $\varphi \in \mathcal{L}_{\rho}(E)$, i.e.

$$
\left\|\sum_{i=1}^{n} V\left(\varphi_{A_{i}}\right)\left(x_{i}\right)\right\| \leq M \rho\left|\sum_{i=1}^{n} \varphi_{A_{i}} x_{i}\right| .
$$

Actually, condition (5.5) is also sufficient for the existence of $U$. Indeed, if (5.5) is fulfilled, it follows that the linear operator $U_{1}(V): S_{E}(\rho) \rightarrow F$ is uniformly continuous, hence it can be extended to an uniformly continuous (and, of course linear) 
operator $U: \overline{S_{E}(\rho)}=\mathcal{L}_{\rho}(E) \rightarrow F$. We have $\Omega(U)=V$, because $\Omega(U)$ and $V$ coincide on all $\varphi_{A}, A \in \mathcal{T}_{\rho}$, hence on the set $S(\rho)$ which is dense in $\mathcal{L}_{\rho}$.

We proved the following fact: A linear and continuous operator $V: \mathcal{L}_{\rho} \rightarrow \mathcal{L}(E, F)$ is $(\rho, E, F)$-natural if and only if it fulfills condition (5.5).

The reader can check condition (5.5) in case $\rho=\|\|_{1}$, with $M=\|V\|_{o}$.

Before going further, let us discuss three particular cases. In the first two, the embedding $\Omega$ is actually a bijection (a linear isomorphism), hence all linear and continuous operators $V \in \mathcal{L}\left(\mathcal{L}_{\rho}, \mathcal{L}(E, F)\right)$ are $(\rho, E, F)$-natural. The third particular case is more interesting and will be studied in more detail.

Case 1. (Finite dimensional case) We shall work with the finite discrete measure space $(T, \mathcal{T}, \mu)=(T, \mathcal{P}(T)$, card), where $T=\{1,2, \ldots, n\}, n \in \mathbf{N}$. The function norm will be assumed to take only finite values, hence $\rho$ is of absolutely continuous type. We shall take $E=K^{p}, p \in \mathbf{N}$ and $F$ will be any Banach space.

Within this framework, we have $\mathcal{L}_{\rho} \equiv K^{n}$ (namely $\varphi \equiv(\varphi(1), \varphi(2), \ldots, \varphi(n))$ for any $\left.\varphi \in \mathcal{L}_{\rho}\right)$ and $\mathcal{L}_{\rho}(E)=\mathcal{L}_{\rho}\left(K^{p}\right) \equiv\left(K^{p}\right)^{n}$ (namely $f \equiv(f(1), f(2), \ldots, f(n))$ for any $f \in \mathcal{L}_{\rho}(E)=\mathcal{L}_{\rho}\left(K^{p}\right)$; writing $f(j)=\left(x_{1 j}, x_{2 j}, \ldots, x_{p j}\right) \in K^{p}$ for any $j=$ $1,2, \ldots, n$, we have $\left.f \equiv\left(x_{u v}\right)_{1 \leq u \leq p, 1 \leq v \leq n} \in K^{p n}\right)$. Define, for any $j=1,2, \ldots, n$, $e_{j} \stackrel{\text { def }}{=} \varphi_{\{j\}} \in \mathcal{L}_{\rho} \equiv K^{n}$ (i.e. $e_{j}=(0, \ldots, 0,1,0, \ldots, 0), 1$ on the $j-t h$ place) and, for any $i=1,2, \ldots, p$, the vector $X_{i}=(0, \ldots, 0,1,0, \ldots, 0)$ ( 1 on the $i$-th place). Then, for a given $V \in \mathcal{L}\left(\mathcal{L}_{\rho}, \mathcal{L}\left(K^{p}, F\right)\right)$, one has, for any $\varphi \equiv(\varphi(1), \varphi(2), \ldots, \varphi(n)) \in \mathcal{L}_{\rho}$ and any $x=\left(x_{1}, x_{2}, \ldots, x_{p}\right) \in K^{p}$, the equality $V(\varphi)(x)=\sum_{i=1}^{p} \sum_{j=1}^{n} x_{i} \varphi(j) V_{i j}$, where $V_{i j}=V\left(e_{j}\right)\left(X_{i}\right) \in F$ for any $1 \leq i \leq p, 1 \leq j \leq n$.

One can prove that, for a given $V$, one has $V=\Omega(U)$, where $U \in \mathcal{L}\left(\mathcal{L}_{\rho}\left(K^{p}\right), F\right)$ is given via $U(f)=\sum_{i=1}^{p} \sum_{j=1}^{n} x_{i j} V_{i j}$ for any $f \equiv\left(x_{i j}\right)_{1 \leq i \leq p, 1 \leq j \leq n}$ in $\mathcal{L}_{\rho}$, hence $V$ is $\left(\rho, K^{p}, F\right)$-natural.

Case 2. $(E=K)$ All operators are natural: $\operatorname{Nat}(\rho, K, F)=\mathcal{L}\left(\mathcal{L}_{\rho}, \mathcal{L}(K, F)\right)$.

In our opinion, the most interesting particular case is the next one.

Case 3. $(F=K)$ Starting with $U \in \mathcal{L}\left(\mathcal{L}_{\rho}(E), K\right)=\mathcal{L}_{\rho}(E)^{\prime}$, we obtain $\Omega(U)=$ $U^{*} \in \mathcal{L}\left(\mathcal{L}_{\rho}, \mathcal{L}(E, K)\right)=\mathcal{L}\left(\mathcal{L}_{\rho}, E^{\prime}\right)$. According to Theorem 4.6, we have $\|U\|_{\rho}=$ \|\|$U \mid \|_{\rho}<\infty$, hence $\mathcal{L} \mathcal{L}\left(\mathcal{L}_{\rho}(E), K\right)=\mathcal{L}_{\rho}(E)^{\prime}$. Consequently, we have the linear and continuous isomorphism $I: \mathcal{L}_{\rho}(E)^{\prime} \rightarrow \mathcal{L} \mathcal{L}\left(\mathcal{L}_{\rho}, E^{\prime}\right)=\Omega\left(\mathcal{L}_{\rho}(E)^{\prime}\right)$, which gives the equality $\operatorname{Nat}(\rho, E, K)=\mathcal{L} \mathcal{L}\left(\mathcal{L}_{\rho}, E^{\prime}\right)$. This equality leads to the following

Theorem 5.5. A linear and continuous operator $V: \mathcal{L}_{\rho} \rightarrow E^{\prime}$ is $(\rho, E, K)$ natural if and only if $\left|\|V \mid\|_{\rho}<\infty\right.$.

From now on, we shall work only with the countable discrete measure space $(T, \mathcal{T}, \mu)=(\mathbf{N}, \mathcal{P}(\mathbf{N})$, card $)$. We shall accept the following

Assumption A. $\mathcal{T}_{\rho}=\{A \subset \mathbf{N} \mid A$ is finite $\}$.

Assumption A is fulfilled e.g. in case $\rho=\|\|_{p}, 1 \leq p<\infty$.

For further purposes, we shall consider, for any $m \in \mathbf{N}$ the function $e_{m} \stackrel{\text { def }}{=}$ $\varphi_{\{m\}}: \mathbf{N} \rightarrow K$, hence $e_{m} \in l_{\rho}$.

It is to be seen that, for any $f \in l_{\rho}(E)$ we have the pointwise equality

$$
f=\sum_{m=1}^{\infty} e_{m} f(m) .
$$

Equality (5.6) is valid also in $l_{\rho}(E)$. Indeed, defining pointwise for any $m \in \mathbf{N}$ the function $u_{m}=\sum_{i=m+1}^{\infty} e_{i} f(i)$, we have $|f| \geq\left|u_{m}\right| \downarrow 0$. Because $\rho$ is of absolutely 
continuous type, it follows that $\rho\left(u_{m}\right) \downarrow 0$. Consequently, for $v_{m}=\sum_{i=1}^{m} e_{i} f(i)=$ $f-u_{m}$, we have $v_{m} \underset{m}{\rightarrow} f$ in $l_{\rho}(E)$, i.e. we have (5.6) in $l_{\rho}(E)$. So, if $U \in \mathcal{L}\left(l_{\rho}(E), F\right)$, we have, for $f \in l_{\rho}(E), U(f)=\sum_{m=1}^{\infty} U\left(e_{m} f(m)\right)$, hence

$$
\|U\|_{\rho}=\sup \left\{\left\|\sum_{m=1}^{\infty} U\left(e_{m} f(m)\right)\right\|\left|f \in l_{\rho}(E), \rho\right| f \mid \leq 1\right\} .
$$

Formula (5.7) should be compared with the formula in the next theorem.

Theorem 5.6. For any $U \in \mathcal{L}\left(l_{\rho}(E), F\right)$ one has

$$
\|U \mid\|_{\rho}=\sup \left\{\sum_{m=1}^{\infty}\left\|U\left(e_{m} f(m)\right)\right\|\left|f \in l_{\rho}(E), \rho\right| f \mid \leq 1\right\} .
$$

Proof. For any $\phi \neq A \in \mathcal{T}_{\rho}$ and any $x \in E:\left\|U\left(\varphi_{A} x\right)\right\|=\left\|U\left(\sum_{n \in A} e_{n} x\right)\right\|=$ $\left\|\sum_{n \in A} U\left(e_{n} x\right)\right\| \leq \sum_{n \in A}\left\|U\left(e_{n} x\right)\right\|$ and this implies, for $f=\sum_{i=1}^{n} \varphi_{A_{i}} x_{i} \in S_{E}(\rho, A)$ : $\sum_{i=1}^{n}\left\|U\left(\varphi_{A_{i}} x_{i}\right)\right\| \leq \sum_{i=1}^{n} \sum_{m \in A_{i}}\left\|U\left(e_{m} x_{i}\right)\right\|=\sum_{i=1}^{n} \sum_{m \in A_{i}}\left\|U\left(e_{m} f(m)\right)\right\|$. We proved that $\sum_{i=1}^{n}\left\|U\left(\varphi_{A_{i}} x_{i}\right)\right\| \leq \sum_{m \in \operatorname{supp}(f)}\left\|U\left(e_{m} f(m)\right)\right\|$ and this implies that $\|U\| \|_{\rho}=$ $\sup \left\{\sum_{m \in \operatorname{supp}(f)}\left\|U\left(e_{m} f(m)\right)\right\| \| f \in S_{E}(\rho, 1)\right\}=\sup \left\{\sum_{m \in \operatorname{supp}(f)}\left\|U\left(e_{m} f(m)\right)\right\| \mid\right.$ $f: \mathbf{N} \rightarrow E, \operatorname{supp}(f)$ is finite and $\rho|f| \leq 1\} \leq M \stackrel{\text { def }}{=} \sup \left\{\sum_{m=1}^{\infty}\left\|U\left(e_{m} f(m)\right)\right\|\right.$ $|f: \mathbf{N} \rightarrow E, \rho| f \mid \leq 1\}$.

It remains to prove that $\left|\|U \mid\|_{\rho} \geq M\right.$.

Case 1. $(M=\infty)$ In the situation when there exists $f: \mathbf{N} \rightarrow E$ such that $\rho|f| \leq 1$ and $\sum_{m=1}^{\infty}\left\|U\left(e_{m} f(m)\right)\right\|=\infty$, take an arbitrary $A>0$. Find $p \in \mathbf{N}$ such that $\sum_{m=1}^{p}\left\|U\left(e_{m} f(m)\right)\right\|>A$, hence $\sum_{m \in \operatorname{supp}(\varphi)}\left\|U\left(e_{m} \varphi(m)\right)\right\|>A$, where $\varphi=\sum_{m=1}^{p} e_{m} f(m)$. Because $\operatorname{supp}(\varphi) \subset\{1,2, \ldots, p\}$ and $\rho|\varphi| \leq 1$, we proved that \|\|$U \mid \|_{\rho}>A$, hence $\|U \mid\|_{\rho}=\infty$, because $A$ is arbitrary. In the situation when $\sum_{m=1}^{\infty}\left\|U\left(e_{m} f(m)\right)\right\|<\infty$ for any $f: \mathbf{N} \rightarrow E$ with $\rho|f| \leq 1$, take again an arbitrary $A>0$ and find $f: \mathbf{N} \rightarrow E$ with $\rho|f| \leq 1$ and $\sum_{m=1}^{\infty}\left\|U\left(e_{m} f(m)\right)\right\|>A+1$. Find $p \in \mathbf{N}$ such that $\sum_{m=1}^{p}\left\|U\left(e_{m} f(m)\right)\right\|>A$, hence $\|U \mid\|_{\rho}>A$ and, again, it follows that $\left|\|U \mid\|_{\rho}=\infty\right.$.

Case 2. $(M<\infty)$ Take an arbitrary $\varepsilon>0$ and find $f: \mathbf{N} \rightarrow E$ such that $\rho|f| \leq 1$ and $\sum_{m=1}^{\infty}\left\|U\left(e_{m} f(m)\right)\right\|>M-\frac{\varepsilon}{2}$. The series $\sum_{m=1}^{\infty}\left\|U\left(e_{m} f(m)\right)\right\|$ being convergent, find a large enough $p \in \mathbf{N}$ such that $\sum_{m=1}^{p}\left\|U\left(e_{m} f(m)\right)\right\|>M-\frac{\varepsilon}{2}-\frac{\varepsilon}{2}=M-\varepsilon$. Using again the function $\varphi=\sum_{m=1}^{p} e_{m} f(m)$, we notice that $\sum_{m \in \operatorname{supp}(\varphi)}\left\|U\left(e_{m} \varphi(m)\right)\right\|>$ $M-\varepsilon$, hence $\left|\|U \mid\|_{\rho}>M-\varepsilon\right.$. Because $\varepsilon$ is arbitrary, we have $|\|U \mid\|_{\rho} \geq M$.

Let us consider in Theorem 5.6 the particular situation when $E=K$, hence $l_{\rho}(E)=l_{\rho}$ and we have a linear and continuous operator $U: l_{\rho} \rightarrow F$. Theorem 5.6 gives ||$|U| \|_{\rho}=\sup \left\{\sum_{m=1}^{\infty}|f(m)|\left\|U\left(e_{m}\right)\right\|\left|f \in l_{\rho}, \rho\right| f \mid \leq 1\right\}$. To say that $\left|\|U \mid\|_{\rho}<\right.$ $\infty$ means $\sup \left\{\sum_{m=1}^{\infty} a_{m}\left\|U\left(e_{m}\right)\right\| \mid a=\left(a_{m}\right)_{m} \in l_{\rho}\right.$, all $\left.a_{m} \geq 0, \rho(a) \leq 1\right\}<\infty$. In case $\rho$ has the Riesz-Fischer property, this means that the sequence $\left(\left\|U\left(e_{m}\right)\right\|\right)_{m}$ is in $l_{\rho^{\prime}}$ (see [3], [24]). We proved

Corollary 5.7. Assume that $\rho$ has the Riesz-Fischer property. Then, for any linear and continuous $U: l_{\rho} \rightarrow F$, one has the equivalence: $\left(\left|\|U \mid\|_{\rho}<\infty\right) \Leftrightarrow\right.$ $\left(\left(U\left(e_{m}\right)\right)_{m} \in l_{\rho^{\prime}}(F)\right)$.

We shall apply Corollary 5.7 to the case when $\rho=\|\|_{p}, 1 \leq p<\infty$ and $F=E^{\prime}$, $E^{\prime}$ being the dual of the Banach space $E$. Then, adapting the proof in $[6]$ for the case of the countable discrete measure space, we have $\left(l^{p}(E)\right)^{\prime} \equiv l^{q}\left(E^{\prime}\right), q$ being the 
conjugate of $p$. Namely, the duality is defined as follows: for any $x=\left(x_{n}\right)_{n} \in l^{p}(E)$ and any $x^{\prime}=\left(x_{n}^{\prime}\right)_{n} \in l^{q}\left(E^{\prime}\right) \equiv\left(l^{p}(E)\right)^{\prime}$, one has $x^{\prime}(x)=\sum_{n=1}^{\infty} x_{n}^{\prime}\left(x_{n}\right)$. It is not necessary for $E^{\prime}$ to have the Radon-Nikodym Property.

Taking into account that for $V: l^{p} \rightarrow E^{\prime}$ to be $(\rho, E, K)$-natural means \|\|$V \mid \|_{\rho}<$ $\infty$ (here $\rho=\|\|_{p}$ ) according to Theorem 5.5 and, using Corollary 5.7, we have

Corollary 5.8. Let $1 \leq p<\infty$ and $E$ a Banach space. Then, a linear and continuous operator $V: l^{p} \rightarrow E^{\prime}$ is $\left(\|\|_{p}, E, K\right)$-natural if and only if $\left(V\left(e_{m}\right)\right)_{m} \in$ $l^{q}\left(E^{\prime}\right)$, where $q$ is the conjugate of $p$.

The framework for the remainder of the paper is the following:

a) We continue to work with the countable discrete measure space, accepting Assumption A.

b) Supplementarily, from now on, we shall take $E=l^{2}$, hence $E^{\prime} \equiv l^{2}$. At the same time, we shall work for $\rho=\|\|_{2}$, hence $\rho^{\prime}=\rho=\|\|_{2}$.

The following identifications are obvious: $\mathcal{L}_{\rho}(E)=l_{\rho}(E)=l^{2}\left(l^{2}\right), \mathcal{L}\left(\mathcal{L}_{\rho}(E), K\right)=$ $\left(\mathcal{L}_{\rho}(E)\right)^{\prime}=\left(l^{2}\left(l^{2}\right)\right)^{\prime} \equiv l^{2}\left(l^{2}\right)$. This means that, for $U \in\left(\mathcal{L}_{\rho}(E)\right)^{\prime}$, one has $U \equiv\left(a^{n}\right)_{n}$ with $a^{n} \in l^{2}$ for any $n$. If $a^{n}=\left(a_{p n}\right)_{p}$ it follows that $U \equiv\left(a_{p n}\right)_{p, n}$ with

$$
\sum_{n=1}^{\infty}\left\|a^{n}\right\|_{2}^{2}=\sum_{n=1}^{\infty} \sum_{p=1}^{\infty}\left|a_{p n}\right|^{2}<\infty
$$

The action of $U$ upon $t=\left(t^{n}\right)_{n} \in \mathcal{L}_{\rho}(E)=l^{2}\left(l^{2}\right)$ is (writing $\left.t^{n}=\left(t_{p n}\right)_{p}\right)$ :

$$
U(t) \stackrel{\text { def }}{=} \sum_{n=1}^{\infty} a^{n}\left(t^{n}\right) \stackrel{\text { def }}{=} \sum_{n=1}^{\infty} \sum_{p=1}^{\infty} a_{p n} t_{p n} .
$$

As a matter of fact, we have

$$
U(t)=\sum_{p=1}^{\infty} \sum_{n=1}^{\infty} a_{p n} t_{p n}
$$

because the double series occuring in (5.9) is absolutely convergent:

$$
\sum_{n=1}^{\infty} \sum_{p=1}^{\infty}\left|a_{p n} t_{p n}\right| \leq \sum_{n=1}^{\infty}\left\|a^{n}\right\|_{2}\left\|t^{n}\right\|_{2} \leq\left(\sum_{n=1}^{\infty}\left\|a^{n}\right\|_{2}^{2}\right)^{\frac{1}{2}} \cdot\left(\sum_{n=1}^{\infty}\left\|t^{n}\right\|_{2}^{2}\right)^{\frac{1}{2}}<\infty .
$$

Continuing with the identifications, we have $\mathcal{L}_{\rho}=l^{2}, \mathcal{L}(E, K)=E^{\prime} \equiv l^{2}$, hence $\mathcal{L}\left(\mathcal{L}_{\rho}, \mathcal{L}(E, K)\right) \equiv \mathcal{L}\left(l^{2}, l^{2}\right)$. Then, considering some $V \in \mathcal{L}(\mathcal{L}, \mathcal{L}(E, K)) \equiv$ $\mathcal{L}\left(l^{2},\left(l^{2}\right)^{\prime}\right) \equiv \mathcal{L}\left(l^{2}, l^{2}\right)$, we apply the general theory and consider the matricial form of $V \equiv\left(v_{p n}\right)_{p, n}$, where the numbers $v_{p n}$ are such that, for any $\varphi=\left(\varphi_{n}\right)_{n} \in \mathcal{L}_{\rho}=l^{2}$, one has $V(\varphi) \equiv y=\left(y_{p}\right)_{p} \in l^{2}$. Namely, for any $p$, one has

$$
y_{p}=\sum_{n=1}^{\infty} v_{p n} \varphi_{n}
$$

In particular, taking $\varphi=e_{n}$ in $(5.11)$, we get $V\left(e_{n}\right) \equiv\left(v_{1 n}, v_{2 n}, \ldots, v_{p n}, \ldots\right)=$ $\left(v_{p n}\right)_{p} \in l^{2}$ for any $n$. Applying Corollary 5.8 we see that $V$ is natural if and only if $\left(V\left(e_{n}\right)\right)_{n} \in l^{2}\left(E^{\prime}\right) \equiv l^{2}\left(l^{2}\right)$, i.e. if and only if

$$
\sum_{n=1}^{\infty} \sum_{p=1}^{\infty}\left|v_{p n}\right|^{2}<\infty
$$


Remark. Relation (5.12) says that $V$ is a Hilbert-Schmidt operator when it is viewed as acting in the standard separable Hilbert space $l^{2}$. We proved that, with this identification, the $\left(\|\|_{2}, l^{2}, K\right)$-natural operators are exactly the Hilbert-Schmidt operators in $\mathcal{L}\left(l^{2}, l^{2}\right)$.

Now, we can describe in a concrete manner the embedding $\Omega: \mathcal{L}\left(\mathcal{L}_{\rho}(E), K\right) \rightarrow$ $\mathcal{L}\left(\mathcal{L}_{\rho}, \mathcal{L}(E, K)\right)$ in this case, i.e., via identification, $\Omega: l^{2}\left(l^{2}\right) \rightarrow \mathcal{L}\left(l^{2}, l^{2}\right)$.

Theorem 5.9. With the previous notations, let $U \equiv\left(a_{p n}\right)_{p, n} \in l^{2}\left(l^{2}\right)$ and $V \equiv$ $\left(v_{p n}\right)_{p, n} \in \mathcal{L}\left(l^{2}, l^{2}\right)$ be in correspondence, i.e. $V=U^{*}=\Omega(U)$. Then, one must have $v_{p n}=a_{p n}$ for any $p$ and $n$.

Proof. We start with an element $V$ in $\Omega\left(l^{2}\left(l^{2}\right)\right) \equiv \operatorname{Nat}\left(\|\|_{2}, l^{2}, K\right)$, hence $V \equiv$ $\left(v_{p n}\right)_{p, n} \in \mathcal{L}\left(l^{2}, l^{2}\right)$, with (5.12) valid. We shall prove that the unique element $U \equiv$ $\left(a_{p n}\right)_{p, n} \in l^{2}\left(l^{2}\right)$ such that $V=\Omega(U)$ is that one given by the fact that $a_{p n}=v_{p n}$ for any $p$ and $n$.

First, we shall see that the postulated construction of $U$ is possible. Indeed, for any $n$, one has $V\left(e_{n}\right)=\left(v_{p n}\right)_{p} \in l^{2}$ and we also have $\sum_{n=1}^{\infty}\left\|V\left(e_{n}\right)\right\|_{2}^{2}<\infty$ (see (5.12)), hence one can take $a^{n}=V\left(e_{n}\right)$ and (5.8) is true for these $a^{n}$. So, one can take $U \equiv\left(a^{n}\right)_{n}=\left(V\left(e_{n}\right)\right)_{n} \in l^{2}\left(l^{2}\right)$. Clearly, $U \equiv\left(v_{p n}\right)_{p, n}$.

It remains to prove that $\Omega(U)=V$, i.e. it remains to prove that, for any $\varphi=$ $\left(\varphi_{1}, \varphi_{2}, \ldots, \varphi_{n}, \ldots\right) \in \mathcal{L}_{\rho}=l^{2}$ and any $x=\left(x_{1}, x_{2}, \ldots, x_{p}, \ldots\right) \in E=l^{2}$, one has $U(\varphi x)=V(\varphi)(x)$.

Clearly, $\varphi x \in \mathcal{L}_{\rho}(E)=l^{2}\left(l^{2}\right)$, with $\varphi x \equiv\left(t^{n}\right)_{n}$, hence $\varphi x \equiv\left(t_{p n}\right)_{p, n}=\left(\varphi_{n} x_{p}\right)_{p, n}$. From (5.10) we obtain for this $U: U(\varphi x)=\sum_{p=1}^{\infty} \sum_{n=1}^{\infty} v_{p n} \varphi_{n} x_{p}$. On the other hand, using (5.11), we get $V(\varphi)(x)=\sum_{p=1}^{\infty} y_{p} x_{p}=\sum_{p=1}^{\infty}\left(\sum_{n=1}^{\infty} v_{p n} \varphi_{n}\right) x_{p}$ and everything is proved. To be very precise, we conclude that, concerning the linear isomorphism $\Omega_{r}: \mathcal{L}\left(\mathcal{L}_{\rho}(E), K\right) \rightarrow \operatorname{Nat}(\rho, E, K)$ considered in this particular case, we proved that, for any $V \equiv\left(v_{p n}\right)_{p, n} \in \operatorname{Nat}(\rho, E, K)$, one has $\Omega_{r}^{-1}(V)=U \equiv\left(v_{p n}\right)_{p, n}$. This implies that, for any $U \equiv\left(a_{p n}\right)_{p, n} \in \mathcal{L}\left(\mathcal{L}_{\rho}(E), K\right)$, one has $\Omega_{r}(U)=\Omega(U)=V \equiv\left(a_{p n}\right)_{p, n}$, thus perfectly describing $\Omega$.

In view of the preceding facts, we shall give some concrete examples.

Example 5.10. (A linear and continuous operator $V: l^{2} \rightarrow\left(l^{2}\right)^{\prime} \equiv l^{2}$ which is not $\left(\|\|_{2}, l^{2}, K\right)$-natural) Take $V \equiv\left(v_{p n}\right)_{p, n}$ (matricial diagonal representation with $v_{p n}=0$, if $p \neq n$ and $v_{p p}=\frac{1}{\sqrt{p}}$ for any $p$ ). Then $\sum_{n=1}^{\infty} \sum_{p=1}^{\infty}\left|v_{p n}\right|^{2}=\sum_{p=1}^{\infty} \frac{1}{p}=\infty$ and $V$ is not natural, in view of (5.12).

Example 5.11. (Linear and continuous operators $V: l^{2} \rightarrow\left(l^{2}\right)^{\prime} \equiv l^{2}$ which are $\left(\|\|_{2}, l^{2}, K\right)$-natural such that either $\|V\|_{\|\|_{2}}<\left|\|V \mid\|_{\|\|_{2}}<\infty\right.$, or $\|V\|_{\|\|_{2}}=$ $\left|\|V \mid\|_{\|\|_{2}}<\infty\right)$ In order to construct such examples, recall that, in case $K=\mathbf{R}$, for a linear operator $W: \mathbf{R}^{2} \rightarrow \mathbf{R}^{2}, W \equiv\left(a_{i j}\right)_{1 \leq i \leq 2,1 \leq j \leq 2}$ and the euclidean norm on $\mathbf{R}^{2}$, one has

$$
\|W\|_{\|\|_{2}}=\|W\|_{o}=\frac{1}{2}\left(\sqrt{\left(a_{11}+a_{22}\right)^{2}+\left(a_{12}-a_{21}\right)^{2}}+\sqrt{\left(a_{11}-a_{22}\right)^{2}+\left(a_{12}+a_{21}\right)^{2}}\right) .
$$

The idea is to consider "two-dimensional" operators $V: l^{2} \rightarrow\left(l^{2}\right)^{\prime} \equiv l^{2}$, namely $V \equiv\left(v_{p n}\right)_{p, n}$ with $v_{p n}=0$ if $p \geq 3$ or $n \geq 3$ (here, we work with $K=\mathbf{R}$, so all $\left.v_{p n} \in \mathbf{R}\right)$. Hence, one has $V \equiv W$, where $W: \mathbf{R}^{2} \rightarrow \mathbf{R}^{2}, W \equiv\left(v_{i j}\right)_{1 \leq i \leq 2,1 \leq j \leq 2}$, which means that, for $\varphi=\left(\varphi_{1}, \varphi_{2}, \ldots, \varphi_{n}, \ldots\right) \in l^{2}$, one has

$$
V(\varphi) \equiv\left(v_{11} \varphi_{1}+v_{12} \varphi_{2}, v_{21} \varphi_{1}+v_{22} \varphi_{2}, 0,0, \ldots\right)
$$

(i.e. $V\left(e_{1}\right) \equiv\left(v_{11}, v_{12}, 0, \ldots\right), V\left(e_{2}\right) \equiv\left(v_{21}, v_{22}, 0, \ldots\right), V\left(e_{n}\right) \equiv(0,0, \ldots)$ for $\left.n \geq 3\right)$. 
It follows that one can compute $\|V\|_{\|\|_{2}}$ using only values $\|V(\varphi)\|_{o}$ for $\varphi \in l^{2}$, $\varphi=\left(\varphi_{1}, \varphi_{2}, \ldots, \varphi_{n}, \ldots\right)$ with $\varphi_{n}=0$, if $n \geq 3$ :

$$
\|V(\varphi)\|_{o}^{2}=\left|v_{11} \varphi_{1}+v_{12} \varphi_{2}+v_{21} \varphi_{1}+v_{22} \varphi_{2}\right|^{2} .
$$

Consequently $\|V\|_{\|\|_{2}}=\|W\|_{o}$.

At the same time, one can see that $\left|\|V \mid\|_{\|\|_{2}}=\sup \left\{\sum_{n=1}^{\infty}\left|x_{n}\right|\left\|V\left(e_{n}\right)\right\|_{o} \mid x=\right.\right.$ $\left(x_{n}\right)_{n} \in l^{2}, x_{n}=0$ for $n \geq 3$ and $\left.\|x\|_{2}=\left(\left|x_{1}\right|^{2}+\left|x_{2}\right|^{2}\right)^{\frac{1}{2}} \leq 1\right\}=\sup \left\{\left|x_{1}\right|\left\|V\left(e_{1}\right)\right\|_{o}+\right.$ $\left.\left|x_{2}\right|\left\|V\left(e_{2}\right)\right\|_{o} \mid x_{1}, x_{2} \in \mathbf{R}, x_{1}^{2}+x_{2}^{2}=1\right\}=\sup \left\{\cos \theta\left\|V\left(e_{1}\right)\right\|_{o}+\sin \theta\left\|V\left(e_{2}\right)\right\|_{o} \mid \theta \in\right.$ $\left.\left[0, \frac{\pi}{2}\right]\right\}=\sqrt{\left\|V\left(e_{1}\right)\right\|_{o}^{2}+\left\|V\left(e_{2}\right)\right\|_{o}^{2}}=\sqrt{v_{11}^{2}+v_{12}^{2}+v_{21}^{2}+v_{22}^{2}}$. Taking $v_{11}=1, v_{22}=2$, $v_{12}=3, v_{21}=4$, we get $\|V\|_{\|\|_{2}}=\frac{1}{2}(\sqrt{10}+\sqrt{50})<\sqrt{30}=\left|\|V \mid\|_{\|\|_{2}}\right.$.

For the equality construction, we use the following equivalences:

$$
\begin{aligned}
& \|V\|_{\|\|_{2}}=\left|\|V \mid\|_{\|\|_{2}} \Leftrightarrow \sqrt{v_{11}^{2}+v_{22}^{2}+v_{12}^{2}+v_{21}^{2}}\right. \\
& =\frac{1}{2}\left(\sqrt{\left(v_{11}+v_{22}\right)^{2}+\left(v_{12}-v_{21}\right)^{2}}+\sqrt{\left(v_{11}-v_{22}\right)^{2}+\left(v_{12}+v_{21}\right)^{2}}\right) \\
& \Leftrightarrow v_{11} v_{22}-v_{12} v_{21}=0 .
\end{aligned}
$$

The last equivalence can be proved by direct elementary computations.

Example 5.12. (showing that, in general, the subspace $\mathcal{L} \mathcal{L}\left(\mathcal{L}_{\rho}(E), F\right)$ is not closed in $\mathcal{L}\left(\mathcal{L}_{\rho}(E), F\right)$ ) Here $\rho=\|\|_{2}, E=K, F=l^{2}$ and we shall exhibit an element $V \in \mathcal{L}\left(l^{2}, l^{2}\right)$ which is in the closure of $\mathcal{L} \mathcal{L}\left(l^{2}, l^{2}\right)$, but is not in $\mathcal{L} \mathcal{L}\left(l^{2}, l^{2}\right)$.

To this end, we consider a sequence $\left(t_{n}\right)_{n} \subset K$ such that $t_{n} \underset{n}{\rightarrow} 0$, but $\left(t_{n}\right)_{n} \notin l^{2}$, e.g. one can take $t_{n}=\frac{1}{\sqrt{n}}$ for any $n$. Let $M=\sup _{n}\left|t_{n}\right|<\infty$. The linear and continuous $V: l^{2} \rightarrow l^{2}$ has diagonal matricial representation $V \equiv\left(v_{p n}\right)_{p, n}$ given as follows: $v_{p n}=0$, if $p \neq n$ and $v_{n n}=t_{n}$ for any $n$. The action of $V: V(x=$ $\left.\left(x_{n}\right)_{n}\right)=\left(t_{n} x_{n}\right)_{n}$, hence $\|V\|_{o} \leq M$. Using the considerations following Theorem 5.6, one has $\left|\|V \mid\|_{\rho}=\sup \left\{\sum_{m=1}^{\infty}|f(m)|\left\|V\left(e_{m}\right)\right\|_{2} \mid f \in l^{2},\|f\|_{2} \leq 1\right\}\right.$ and this gives \|\|$V \mid\left\|_{\rho}=\rho^{\prime}(u)=\right\| u \|_{2}$, where $u=\left(\left\|V\left(e_{m}\right)\right\|_{2}\right)_{m}$. Because $V\left(e_{m}\right)=t_{m} e_{m}$, we have $\left\|V\left(e_{m}\right)\right\|_{2}=\left|t_{m}\right|$, hence $u \notin l^{2}$, consequently $\left|\|V \mid\|_{\rho}=\infty\right.$ and $V \notin \mathcal{L} \mathcal{L}\left(l^{2}, l^{2}\right)$.

Finally, we construct a sequence $\left(V_{m}\right)_{m} \subset \mathcal{L} \mathcal{L}\left(l^{2}, l^{2}\right)$ such that $V_{m} \underset{m}{\rightarrow} V$ in $\mathcal{L}\left(l^{2}, l^{2}\right)$. Namely $V_{m}: l^{2} \rightarrow l^{2}$ will have diagonal matricial representation, for any $m$, as follows: $V_{m} \equiv\left(v_{p n}^{m}\right)_{p, n}$, where $v_{p n}^{m}=0$, if $p \neq n, v_{n n}^{m}=t_{n}$, if $n \leq m$ and $v_{n n}^{m}=0$, if $n \geq m+1$. Because $V_{m}\left(e_{n}\right)=t_{n} e_{n}$, if $n \leq m$ and $V_{m}\left(e_{n}\right)=0$, if $n \geq m+1$, it follows that $\left(\left\|V_{m}\left(e_{n}\right)\right\|_{2}\right)_{n} \in l^{2}$ and all $V_{m} \in \mathcal{L} \mathcal{L}\left(l^{2}, l^{2}\right)$. We have $V_{m} \underset{m}{\rightarrow} V$ in $\mathcal{L}\left(l^{2}, l^{2}\right)$, because the diagonal matricial representation of $V-V_{m}$ gives $\left\|V-V_{m}\right\|_{o} \leq \sup _{n \geq m+1}\left|t_{n}\right| \underset{m}{\rightarrow} 0$.

Remark. Theorem 5.5 and this example show that, in general, the subspace of $(\rho, E, K)$-natural operators is not closed in $\mathcal{L}\left(\mathcal{L}_{\rho}, E^{\prime}\right)$.

Acknowledgemets. The authors are very much indebted to the editors and to the anonymous referee for their kind and most valuable suggestions, which improved a lot the quality of the present paper.

\section{References}

[1] Almeida, A.: Inversion of the Riesz potential operator on Lebesgue spaces with variable exponent. - Fract. Calc. Appl. Anal. 6, 2003, 311-327. 
[2] Calabuig, J. M., J. Rodríguez, and E. A. SÁnchez PÉrez: Multiplication operators in Köthe-Bochner spaces. - J. Math. Anal. Appl. 373, 2011, 316-321.

[3] Chiţescu, I.: Function spaces. - Ed. Şt. Encicl., Bucharest, 1983 (in Romanian).

[4] Chiţescu, I., R.-C. Sfetcu, and O. Cojocaru: Köthe-Bochner spaces: general properties. - Bull. Braz. Math. Soc. (N.S.), 2018, https://doi.org/10.1007/s00574-018-0101-0.

[5] Cruz-Uribe, D., A. Fiorenza, J. M. Martell, and C. PÉrez: The boundedness of classical operators on variable $L^{p}$ spaces. - Ann. Acad. Sci. Fenn. Math. 31, 2006, 239-264.

[6] Diestel, J., and J. J. Uhl JR.: Vector measures. - Mathematical Surveys 15, Amer. Math. Soc., Providence, Rhode Island, 1977.

[7] Dinculeanu, N.: Vector measures. - Pergamon Press, New York, 1967.

[8] Dunford, N., and J. T. Schwartz: Linear operators. Part I. - Interscience Publishers, New York, 1957.

[9] Gretsky, N.E., and J. J. Uhl JR.: Bounded linear operators on Banach function spaces of vector-valued functions. - Trans. Amer. Math. Soc. 167, 1972, 263-277.

[10] Gretsky, N.E., and J. J. UhL JR.: Bounded linear operators into vector-valued Banach function spaces. - Indag. Math. (Proceedings) 77, 1974, 457-462.

[11] Halmos, P. R.: Measure theory. - D. Van Nostrand Company, Inc., 11th printing, 1966.

[12] Köтнe, G.: Neubergründung der Theorie der vollkommenen Räume. - Math. Nachr. 4, 1951, 70-80.

[13] Köthe, G., and O. Toeplitz: Lineare Räume mit unendlichvielen Koordinaten und Ringe unendlicher Matrizen. - Journal de Crelle 171, 1934, 193-226.

[14] Lin, P.-K.: Köthe-Bochner function spaces. - Springer Science+Business Media, LLC, 2004.

[15] Luxemburg, W. A. J.: Banach function spaces. - Thesis, Delft Institute of Technology, Assen, Netherlands, 1955.

[16] Luxemburg, W. A. J., and A. C. ZaAnen: Notes on Banach function spaces. - Indag. Math. Note I, 1963 - Note XVI, 1965.

[17] Luxemburg, W. A. J., and A. C. ZaAnen: Riesz spaces. Volume 1. - North Holland Publishing Company, Amsterdam, London, 1971.

[18] Maldonado, M., and J. Prada: Weighted shift operators on Köthe spaces. - Math. Nachr. 279, 2006, 188-197.

[19] Mykhaylyuk, V. V., and M. M. Popov: On sums of narrow operators on Köthe function spaces. - J. Math. Anal. Appl. 404, 2013, 554-561.

[20] NekvindA, A.: Hardy-Littlewood maximal operator on $L^{p(x)}\left(\mathbf{R}^{n}\right)$. - Math. Inequal. Appl. 7, 2004, 255-265.

[21] SÁnchez PÉrez, E. A.: Asymptotic domination of operators on Köthe function spaces and convergence of sequences. - Math. Nachr. 279, 2006, 1709-1722.

[22] Wu, X.: Maximal distributional chaos of weighted shift operators on Köthe sequence spaces. - Czech. Math. J. 64, 2014, 105-114.

[23] Wu, X., and P. ZHU: Li-Yorke chaos of backward shift operators on Köthe sequence spaces. - Topology Appl. 160, 2013, 924-929.

[24] ZaAnen, A. C.: Integration. - North Holland, Amsterdam, 1967.

[25] ZaAnen, A. C.: Riesz spaces. Volume 2. - North Holland Publishing Company, Amsterdam, New York, Oxford, 1983.

Received 26 September 2018 • Accepted 10 January 2019 